\title{
Genome analyses of four Wolbachia strains and associated mitochondria of Rhagoletis cerasi expose cumulative modularity of cytoplasmic incompatibility factors and cytoplasmic hitchhiking across host populations
}

Jennifer L. Morrow and Markus Riegler

\begin{abstract}
Background: The endosymbiont Wolbachia can manipulate arthropod reproduction and invade host populations by inducing cytoplasmic incompatibility $(\mathrm{Cl})$. Some host species are coinfected with multiple Wolbachia strains which may have sequentially invaded host populations by expressing different types of modular $\mathrm{Cl}$ factor (cif) genes. The tephritid fruit fly Rhagoletis cerasi is a model for $\mathrm{Cl}$ and Wolbachia population dynamics. It is associated with at least four Wolbachia strains in various combinations, with demonstrated (wCer2, wCer4), predicted (wCer1) or unknown (wCer5) Cl phenotypes.

Results: We sequenced and assembled the draft genomes of the Wolbachia strains wCer1, wCer4 and wCer5, and compared these with the previously sequenced genome of wCer 2 which currently invades $R$. cerasi populations. We found complete cif gene pairs in all strains: four pairs in wCer2 (three Type I; one Type V), two pairs in wCer1 (both Type I) and wCer4 (one Type I; one Type V), and one pair in wCer5 (Type IV). Wolbachia genome variant analyses across geographically and genetically distant host populations revealed the largest diversity of single nucleotide polymorphisms (SNPs) in wCer5, followed by wCer1 and then wCer2, indicative of their different lengths of host associations. Furthermore, mitogenome analyses of the Wolbachia genome-sequenced individuals in combination with SNP data from six European countries revealed polymorphic mitogenome sites that displayed reduced diversity in individuals infected with wCer2 compared to those without.
\end{abstract}

\footnotetext{
* Correspondence: m.riegler@westernsydney.edu.au

Hawkesbury Institute for the Environment, Western Sydney University, Locked Bag 1797, Penrith, NSW 2751, Australia
}

(c) The Author(s). 2021 Open Access This article is licensed under a Creative Commons Attribution 4.0 International License, which permits use, sharing, adaptation, distribution and reproduction in any medium or format, as long as you give appropriate credit to the original author(s) and the source, provide a link to the Creative Commons licence, and indicate if changes were made. The images or other third party material in this article are included in the article's Creative Commons licence, unless indicated otherwise in a credit line to the material. If material is not included in the article's Creative Commons licence and your intended use is not permitted by statutory regulation or exceeds the permitted use, you will need to obtain permission directly from the copyright holder. To view a copy of this licence, visit http://creativecommons.org/licenses/by/4.0/ The Creative Commons Public Domain Dedication waiver (http://creativecommons.org/publicdomain/zero/1.0/) applies to the data made available in this article, unless otherwise stated in a credit line to the data. 
Conclusions: Coinfections with Wolbachia are common in arthropods and affect options for Wolbachia-based management strategies of pest and vector species already infected by Wolbachia. Our analyses of Wolbachia genomes of a host naturally coinfected by several strains unravelled signatures of the evolutionary dynamics in both Wolbachia and host mitochondrial genomes as a consequence of repeated invasions. Invasion of already infected populations by new Wolbachia strains requires new sets of functionally different cif genes and thereby may select for a cumulative modularity of cif gene diversity in invading strains. Furthermore, we demonstrated at the mitogenomic scale that repeated Cl-driven Wolbachia invasions of hosts result in reduced mitochondrial diversity and hitchhiking effects. Already resident Wolbachia strains may experience similar cytoplasmic hitchhiking effects caused by the invading Wolbachia strain.

Keywords: Reproductive parasite, Haplotypes, Invasion dynamics, cifA, cifB, Rhagoletis cerasi, Ceratitis capitata

\section{Background}

Maternally inherited endosymbiotic Wolbachia bacteria (Alphaproteobacteria) of arthropods can affect host reproduction and fitness, including host immunity, in a multitude of ways $[1,2]$, and consequently, influence the diversity of mitochondria [3] and possibly other cytoplasmic and non-cytoplasmic factors [4]. One commonly reported reproductive manipulation by Wolbachia is cytoplasmic incompatibility (CI). In its simplest form, $\mathrm{CI}$ involves a modification to the sperm of a $\mathrm{Wol}$ bachia-infected male which is not rescued after fertilisation of an egg from an uninfected female, resulting in embryonic mortality [5]. In contrast, an infected female transmits Wolbachia to her eggs thereby restoring successful embryonic development. Other reproductive manipulations by Wolbachia are male killing (MK), thelytokous parthenogenesis and feminisation [1]. While reproductive manipulations such as $\mathrm{CI}$ and MK bestow a reproductive advantage on Wolbachia-infected females in populations of mixed infection status, other Wolbachia effects can also contribute to host fitness. These can be beneficial such as nutrient provisioning $[6,7]$, increased fecundity $[8]$ and pathogen protection $[9,10]$; or costly such as reduced fecundity [11] and shortened life span [12].

A large number of host species are associated with more than one CI-inducing Wolbachia strain [13-15], indicating that some have experienced either consecutive or simultaneous invasions by multiple Wolbachia strains. These can occur as coinfections in individuals or as different infection types within and between populations and may result in complex interactions of $\mathrm{CI}$ and host fitness effects. For example, two or more CI-inducing Wolbachia strains co-infecting individuals of a species can produce patterns of unidirectional CI when one of these CI-inducing strains is missing in females, whereas males without this strain are still compatible with either type of infected females [16]. The rarer observed form is bidirectional CI which occurs when two or more CIinducing Wolbachia strains do not co-occur in the same individuals, and this can result in bidirectional reproductive barriers between differently infected populations contributing to reproductive isolation and speciation processes [17-19].

The genetic basis of $\mathrm{CI}$ has recently been uncovered with the finding that Wolbachia induces and rescues CI through the dual expression of the cytoplasmic incompatibility factor genes cifA and cifB located in Wolbachia prophage regions $[20,21]$. A proposed two-by-one model predicts that both cifA and cifB induce $\mathrm{CI}$, whereas cifA only is required for rescue [22]. Sequence similarity analyses have revealed a large diversity of cif genes in Wolbachia genomes with zero to four complete pairs of cif genes common in strains [23-25]; but up to seven cif complexes have been found in some strains which also include unpaired, partial or non-functional cif genes [26]. The diversity of cifA and cifB gene products are classified as Type I to Type V based on amino acid similarity in functional domains [20, 23, 27]. The CI phenotype has been demonstrated by transgenic expression for Type I, Type II and Type IV cif genes [20, 21, 28], while $w$ No, with a single Type III cif gene pair, and $w$ Stri, with multiple Type V cif gene pairs, both also induce CI [27, 29-31]. Closely related cif genes within a type tend to be compatible; this applies particularly to cifA genes, whereas more variability in cifB genes correlates with phenotypic variation $[28,32]$. However, divergence across the different types results in incompatibility between cif genes of Type I (e.g. in wMel and wPip), Type II (e.g. in $w$ Ri) and Type IV (e.g. in $w$ Pip) $[20,21$, 28]. Therefore, the diversity and modularity of cif genes found in and across Wolbachia genomes may explain the complexity of CI interactions seen between Wolbachia strains, including the expression of bidirectional CI between strains with different types and/or numbers of cif genes even if these strains have similar multi locus sequence typing (MLST) profiles [33].

With their seminal paper on the incompatible populations of the European cherry fruit fly, Rhagoletis cerasi (Tephritidae), Boller and Bush [34] unknowingly established a key study system for Wolbachia population dynamics and CI. Their findings fit the model of 
unidirectional CI expressed between southern and northern populations of this species [35], which was hypothesised to be induced by intracellular Rickettsialike microorganisms identified by electron microscopy [36]. Later, two Wolbachia strains, wCer1 and $w$ Cer2 were discovered that existed as either single $w \operatorname{Cer} 1$ infections in all individuals of all populations, or coinfections with $w$ Cer2 in almost all individuals of southern populations, with individuals of transitional populations between the two population blocks displaying either of the two infection types [13, 37]. The strains' geographic distribution correlated with the patterns of the previously reported unidirectional CI thereby indicating that $w$ Cer2 induces CI between these populations which $w$ Cer1 did not rescue [13]. The interactions of $R$. cerasi with Wolbachia were further complicated by the discovery of three other strains, usually found at lower titres than $w$ Cer1 and $w$ Cer2 [14]. Of the five strains, $w$ Cer1, $w$ Cer2, $w$ Cer4 (all supergroup A strains) and $w$ Cer5 (a supergroup B strain) were characterised by MLST [38], however, the existence of the strain $w$ Cer3 was unclear because it was only ever detected as a wsp gene sequence by molecular cloning of wsp PCR amplicons, and consisted of a sequence which was a recombinant between $w s p$ of $w$ Cer2 and $w$ Cer5 [14]. Across the host range $w$ Cer3 3 was rare, whereas the prevalences of $w$ Cer4 $(60-78 \%)$ and $w$ Cer5 $(3-100 \%)$ were moderate and without any clear patterns [14] when contrasted with the distribution of $w$ Cer1 and $w$ Cer2 $[13,37]$. Therefore, coinfections of $R$. cerasi individuals can include all possible combinations of $w$ Cer1 with one, two or three of the strains $w$ Cer2, $w$ Cer4 4 and $w$ Cer5 $[14,38]$.

While there is strong indirect and correlative evidence for the CI phenotype of $w$ Cer2 in $R$. cerasi because of the distribution of Wolbachia strains and CI patterns $[13,14]$, the direct testing of CI phenotypes of the $\mathrm{Wol}$ bachia strains in this host species by crossing experiments between individuals of defined infection status is difficult due to its strict univoltine life cycle with an obligate pupal diapause [39] and complex laboratory rearing protocols [40]. However, the capacity of $w C e r 2$ and $w$ Cer4 to induce and rescue CI was demonstrated in a series of experiments involving transfer into novel host species by microinjections: for $w$ Cer2 this resulted in expression of moderate CI in Drosophila simulans [41], and complete CI in the Mediterranean fruit fly Ceratitis capitata [42] and the olive fly Bactrocera oleae [43]; for $w$ Cer4 it resulted in the expression of complete CI in C. capitata [42]. Moreover, whole genome sequencing of $w C e r 2$ from infected $R$. cerasi, $D$. simulans and $C$. capitata revealed that the $w$ Cer2 genome contains three pairs of Type I cif genes and one pair of Type V cif genes [24, 44].
Furthermore, there is a tight linkage of $w$ Cer2 with a particular mitochondrial haplotype of $R$. cerasi, denoted haplotype 2 (HT2) which differs by a single nucleotide polymorphism (SNP; a synonymous third codon transition) in the mitochondrial cytochrome oxidase subunit I (COI) gene from HT1 found in individuals lacking $w$ Cer2 [45]. This suggests mitochondrial hitchhiking of HT2 with a recent and still ongoing CI-driven invasion of host populations by $w$ Cer2 $[13,37,46,47]$, and this was expected as a consequence of an ongoing Wolbachia invasion [3]. Besides this pattern of mitochondrial hitchhiking, it also appears that overall $R$. cerasi has very low mitochondrial DNA diversity (i.e. just two COI haplotypes) which may be indicative of several consecutive selective sweeps of mitochondrial genomes which has resulted in the elimination of mitogenome diversity in this species because of repeated invasions by Wolbachia. Specifically, $w$ Cer1 which is fixed across $R$. cerasi populations is tightly linked with HT1, and may have invaded this host by $\mathrm{CI}$, prior to the host's invasion by $w$ Cer2 [45]. A non-exclusive alternative reason for its high prevalence could be that $w$ Cer1 provides a fitness benefit to the host, but this could still cause a selective sweep of an associated haplotype [3]. Furthermore, while $w$ Cer4 causes $\mathrm{CI}$ in the novel host $C$. capitata [42], the CI potential and invasion history of $w$ Cer 4 and $w$ Cer 5 in their native host $R$. cerasi remain unknown. These strains may also have invaded the host by $\mathrm{CI}$, prior to the invasions by $w$ Cer 2 and $w$ Cer1. Alternatively, they may have other mechanisms by which they have invaded and are maintained in host populations, and this could include MK [48, 49]. A MK candidate gene has recently been identified within the Wolbachia prophage WOMelB region of $D$. melanogaster in the vicinity of cifA and cifB and named WO-mediated killing (wmk). It has six additional orthologues in the $w \mathrm{Mel}$ genome, but wmk is almost identical to a single homologue in $w$ Rec, the MK Wolbachia strain of Drosophila recens. Wmk can cause MK when highly expressed in transgenic $D$. melanogaster, while wmk and its orthologues in wMel do not have this effect [50].

Here we sequenced and analysed the genomes of $w$ Cer $1, w$ Cer 4 and $w$ Cer5, and compared these with the previously sequenced $w C e r 2$ genome [24] with a particular focus on their cif and wmk gene repertoires. We expected to find full sets of diverse cif genes: for $w$ Cer4 because of its $\mathrm{CI}$ expression in the novel host $C$. capitata, and for $w$ Cer1 because of its very high prevalence and mitochondrial diversity patterns in the native host $R$. cerasi. Furthermore, we expected different (and potentially fewer) cif gene pairs and types in $w$ Cer $1, w$ Cer 4 and $w$ Cer 5 than found in $w$ Cer 2 which has more recently infected this host species. This is because for any $\mathrm{CI}$ drive to occur, newly arriving Wolbachia strains 
would require cif genes that are novel to a host species already infected by other resident Wolbachia strains. We did not have prior expectations with regard to the presence of $w m k$ genes because MK in $R$. cerasi has not been reported. Furthermore, the expression of MK also strongly depends on the host genotype [51, 52].

Moreover, we explored the $R$. cerasi mitogenomes of the individuals from which we obtained the Wolbachia genomes and used these mitogenomic data to guide the extraction and analysis of additional mitochondrial and Wolbachia SNP data, Wolbachia infection status and geographic information from a published double digest restriction-site associated DNA sequencing (ddRADseq) dataset of $192 R$. cerasi individuals from six European countries [53]. We expected that greater mitochondrial haplotype variation is found in HT1 individuals lacking $w$ Cer2 because these would not have experienced the selective sweep of HT2 caused by the $w$ Cer2 invasion. Similarly, we expected to find greater SNP variation within the genomes of Wolbachia strains (i.e. Wolbachia strain variants) that have a longer association with $R$. cerasi because their genomes would have had more time to acquire new mutations since host invasion. They could also have experienced cytoplasmic hitchhiking effects similar to the ones experienced by mitochondrial genomes due to the $w$ Cer2 invasion. Finally, we combined these three approaches of data analyses, (i) cif gene diversity and module number, (ii) mitogenome variant analyses and (iii) Wolbachia strain variant analyses, to infer the historical order of Wolbachia strain invasions in $R$. cerasi. We anticipated finding confirmation that $w$ Cer2 is the most recent invader in this host species, following the prior invasions by $w$ Cer1 and the other strains.

\section{Results}

Gene content of the three Wolbachia genomes wCer1, wCer4 and wCer5

Genome amplification libraries of three $R$. cerasi fieldcollected individuals, one each from three geographically distant and genetically diverged populations, Austria (RcerAS), Hungary (RcerHB) and Italy (RcerIZ), and one individual of the microinjected C. capitata laboratory population (Ccap10.3) were sequenced to acquire the genomes of four Wolbachia strains and the R. cerasi hosts' mitochondria (Fig. 1). Reads from each library were initially mapped to the MLST markers of each of the strains $w$ Cer $1, w$ Cer2, $w$ Cer 4 and $w$ Cer 5 to confirm the infection status of each individual used for library preparation (Table 1). RcerHB harboured $w$ Cer1 only, and Ccap10.3 harboured $w$ Cer4 only; RcerIZ was coinfected with $w$ Cer1 and $w$ Cer5, and RcerAS was coinfected with

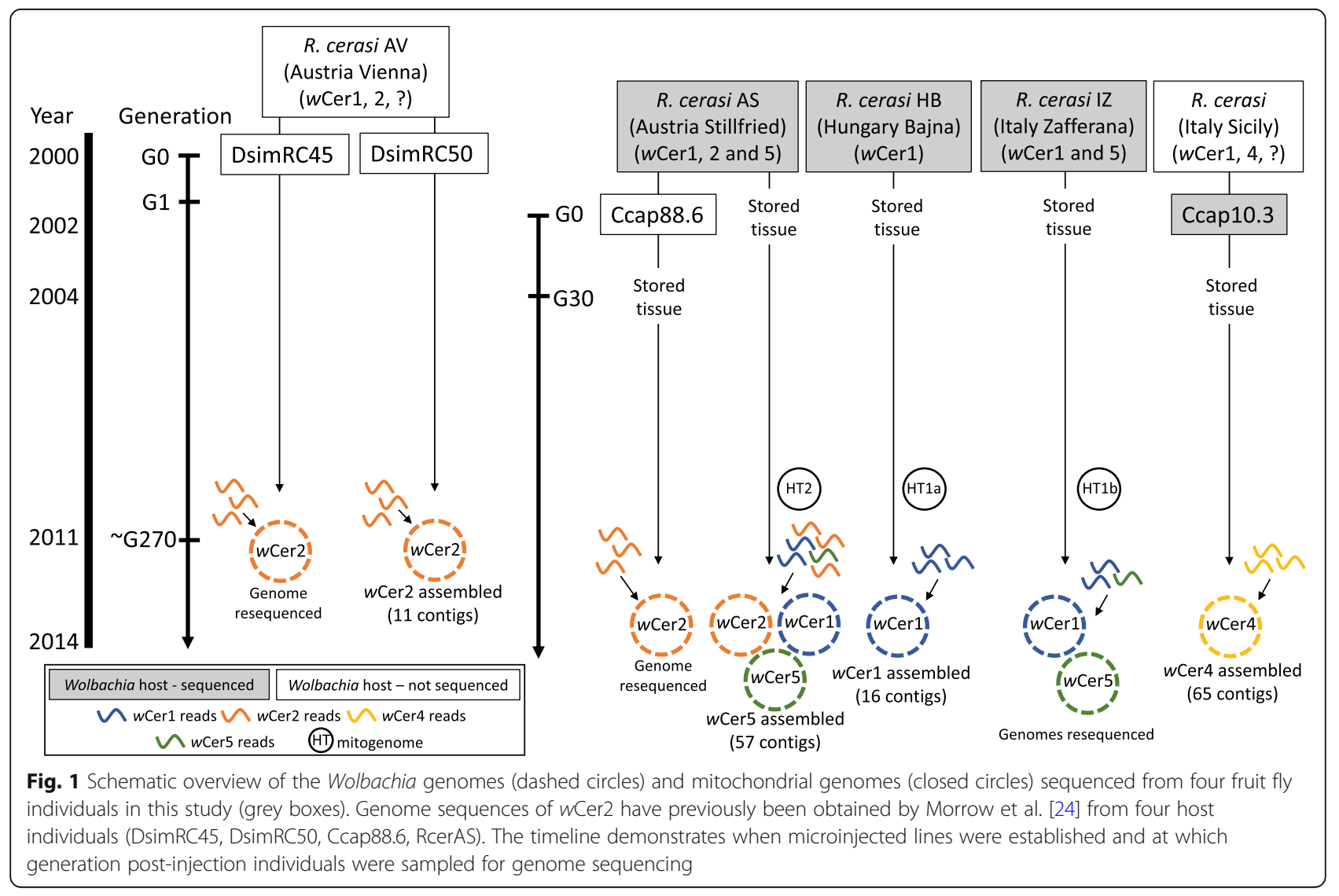


Table 1 The mapping coverage of mitogenomes and genomes of Wolbachia strains obtained from three Rhagoletis cerasi individuals and one Ceratitis capitata individual. Genomes of wCer1, wCer2 and wCer4 were assembled from libraries of individuals which only had one strain. Then these reference genomes were used to competitively map reads of RcerAS to wCer1, wCer2 and wCer5, and reads of RcerlZ to wCer1 and wCer5; all other libraries (and sampled libraries) were mapped to a single Wolbachia strain. Subsampling of total reads was selectively applied to normalise the number of mapped reads for comparison between libraries. Mapping parameters were $97 \%$ similarity over $97 \%$ length, keeping only properly paired reads

\begin{tabular}{|c|c|c|c|c|}
\hline Host Species & Rhagoletis cerasi & & & C. capitata \\
\hline Population & $\begin{array}{l}\text { RcerHB; Bajna, } \\
\text { Hungary }\end{array}$ & $\begin{array}{l}\text { RcerlZ; Zafferana, } \\
\text { Italy }\end{array}$ & $\begin{array}{l}\text { RcerAS; Stillfried, } \\
\text { Austria }\end{array}$ & $\begin{array}{l}\text { Ccap10.3 } \\
\text { (WolMed S10.3) }\end{array}$ \\
\hline Tissue source (DNA extraction) & $\begin{array}{l}\text { single pupa } \\
\text { (whole individual) }\end{array}$ & $\begin{array}{l}\text { single larva } \\
\text { (whole individual) }\end{array}$ & $\begin{array}{l}\text { single pupa } \\
\text { (whole individual) }\end{array}$ & $\begin{array}{l}\text { single adult } \\
\text { (abdomen) }\end{array}$ \\
\hline $\begin{array}{l}\text { Wolbachia strain detected by } \\
\text { MLST markers }\end{array}$ & wCer1 & wCer1; wCer5 & wCer1; wCer2; wCer5 & wCer4 \\
\hline No. of paired reads after $Q C$ & $125,415,852$ & $147,147,772$ & $131,385,710$ & $130,835,872$ \\
\hline $\begin{array}{l}\text { No. paired reads mapped to } \\
\text { mtDNA (full library) }\end{array}$ & $19,390,560$ & $1,053,116$ & $39,939,364$ & $31,839,758$ \\
\hline Percentage mapped to mtDNA & $15.46 \%$ & $0.72 \%$ & $30.40 \%$ & $24.34 \%$ \\
\hline $\begin{array}{l}\text { No. paired reads mapped to } \\
\text { mtDNA (no. subsampled) }\end{array}$ & $81,548(530,000)$ & 85,244 (12million) & $81,608(270,000)$ & na \\
\hline Percentage mapped to mtDNA & $15.46 \%$ & $0.71 \% \%$ & $30.23 \% \%$ & na \\
\hline $\begin{array}{l}\text { No. paired reads mapped to } \\
\text { wCer1 (full library) }\end{array}$ & $3,586,116$ & 258,194 & $1,411,170$ & na \\
\hline Percentage mapped to $w C e r 1$ & $2.86 \%$ & $0.18 \%$ & $1.08 \%$ & na \\
\hline $\begin{array}{l}\text { No. paired reads mapped to } \\
w C e r 1 \text { (no. subsampled) }\end{array}$ & 286,212 (10million) & na & 278,582 (26million) & na \\
\hline Percentage mapped to $w C e r 1$ & $2.86 \%$ & na & $1.07 \%$ & na \\
\hline $\begin{array}{l}\text { No. paired reads mapped to } \\
w \text { Cer2 (full library) }\end{array}$ & na & na & $2,561,484$ & na \\
\hline Percentage mapped to $w C e r 2$ & na & na & $1.95 \%$ & na \\
\hline $\begin{array}{l}\text { No. paired reads mapped to } \\
\text { wCer2 (no. subsampled) }\end{array}$ & na & na & 1,697,166 (87million) & na \\
\hline Percentage mapped to $w C e r 2$ & na & na & $1.95 \%$ & na \\
\hline $\begin{array}{l}\text { No. paired reads mapped to } \\
\text { wCer4 (full library) }\end{array}$ & na & na & na & $1,701,414$ \\
\hline Percentage mapped to wCer4 & na & na & na & $1.30 \%$ \\
\hline $\begin{array}{l}\text { No. paired reads mapped to } \\
w \text { Cer5 (full library) }\end{array}$ & na & 51,402 & 191,892 & na \\
\hline Percentage mapped to wCer5 & na & $0.03 \%$ & $0.15 \%$ & na \\
\hline
\end{tabular}

$w$ Cer1, $w$ Cer2 and $w$ Cer5. None of the libraries contained the recombinant $w s p$ gene of $w$ Cer3.

The three new Wolbachia draft genomes presented here were not closed but deemed to be near complete by BUSCO analysis (Table 2). The BUSCO score for the $w$ Cer1 genome (16 contigs) was $82.8 \%$, the $w$ Cer 4 genome (65 contigs) was $83.3 \%$, and the $w$ Cer5 genome (57 contigs) was $81 \%$, and all three were comparable to complete Wolbachia genomes that also had BUSCO scores between 81.4 and $83.7 \%$.

OrthoFinder assigned 24,268 coding genes $(97.6 \%$ of total 24,859 coding genes) of 19 Wolbachia genomes to 1373 orthogroups. Of these, 738 orthogroups were present in all genomes, and 664 consisted entirely of single-copy genes (Additional File 1). Testing for recombination using PhiPack identified 408 orthogroups that were excluded, with 256 orthogroups remaining. Testing of monophyly of the remaining genes for supergroup A and $B$ strains (Table 2) found no additional genes that should be excluded due to polyphyly. A maximum likelihood tree was built on this set of 256 orthologous genes of 19 Wolbachia genomes and included 183,819 nucleotide sites of which 25,996 were parsimony-informative sites (Fig. 2). This analysis confirmed the assignment of $w$ Cer 1 and $w$ Cer4 (along with $w$ Cer2) into supergroup A and $w$ Cer5 into supergroup B.

Visualisation of the intersection of orthologous genes using the UpSet graph also supported the strong 
Table 2 Genome characteristics, number of scaffolds and BUSCO scores (genome completeness) for wCer1, wCer4, wCer5 (in bold) and the genomes of 16 reference strains (ordered by supergroups A and B, and then alphabetically)

\begin{tabular}{|c|c|c|c|c|c|c|c|c|c|c|}
\hline Strain & Host & Supergroup & Accession No. & $\begin{array}{l}\text { Genome } \\
\text { size (bp) }\end{array}$ & $\begin{array}{l}\text { Number of } \\
\text { scaffolds }\end{array}$ & GC\% & $\begin{array}{l}\text { Predicted } \\
\text { CDSs }\end{array}$ & tRNAs & rRNAs & $\begin{array}{l}\text { BUSCO } \\
\text { score }\end{array}$ \\
\hline wAu & Drosophila simulans & A & LK055284 & $1,268,461$ & 1 & 35.2 & 1276 & 34 & $\begin{array}{l}1 \text { of } \\
\text { each }\end{array}$ & $\begin{array}{l}185 \\
(83.7 \%)\end{array}$ \\
\hline wCauA & Carposina sasakii & A & NZ_CP041215 & $1,449,344$ & 1 & 35.0 & 1442 & 34 & $\begin{array}{l}1 \text { of } \\
\text { each }\end{array}$ & $\begin{array}{l}184 \\
(83.3 \%)\end{array}$ \\
\hline$w C e r 1$ & $\begin{array}{l}\text { Rhagoletis cerasi } \\
\text { (RcerHB) }\end{array}$ & A & JADCNC01000000 & $1,255,676$ & 16 & 35.2 & 1196 & 34 & $\begin{array}{l}1 \text { of } \\
\text { each }\end{array}$ & $\begin{array}{l}183 \\
(82.8 \%)\end{array}$ \\
\hline$w$ Cer2 & $\begin{array}{l}\text { Drosophila simulans } \\
\text { (DsimRC50) }\end{array}$ & A & SOZK01000000 & $1,325,568$ & 11 & 35.2 & 1259 & 34 & $\begin{array}{l}1 \text { of } \\
\text { each }\end{array}$ & $\begin{array}{l}184 \\
(83.3 \%)\end{array}$ \\
\hline$w C e r 4$ & $\begin{array}{l}\text { C. capitata } \\
\text { (Ccap10.3) }\end{array}$ & A & JADCND01000000 & $1,239,646$ & 65 & 35.1 & 1214 & 34 & $\begin{array}{l}1 \text { of } \\
\text { each }\end{array}$ & $\begin{array}{l}184 \\
(83.3 \%)\end{array}$ \\
\hline$w \mathrm{Ha}$ & Drosophila simulans & A & NC_021089 & $1,295,804$ & 1 & 35.1 & 1235 & 34 & $\begin{array}{l}1 \text { of } \\
\text { each }\end{array}$ & $\begin{array}{l}183 \\
(82.8 \%)\end{array}$ \\
\hline whr & $\begin{array}{l}\text { Haematobia irritans } \\
\text { irritans }\end{array}$ & A & NZ_CP037426 & $1,352,354$ & 1 & 35.3 & 1439 & 34 & $\begin{array}{l}1 \text { of } \\
\text { each }\end{array}$ & $\begin{array}{l}184 \\
(83.3 \%)\end{array}$ \\
\hline wMeg & $\begin{array}{l}\text { Chrysomya } \\
\text { megacephala }\end{array}$ & A & NZ_CP021120 & $1,376,868$ & 1 & 34.0 & 1298 & 34 & $\begin{array}{l}1 \text { of } \\
\text { each }\end{array}$ & $\begin{array}{l}182 \\
(82.4 \%)\end{array}$ \\
\hline wMel & $\begin{array}{l}\text { Drosophila } \\
\text { melanogaster }\end{array}$ & A & NC_002978 & 1267,782 & 1 & 35.2 & 1271 & 34 & $\begin{array}{l}1 \text { of } \\
\text { each }\end{array}$ & $\begin{array}{l}184 \\
(83.3 \%)\end{array}$ \\
\hline wec & Drosophila recens & A & NZ_JQAM01000000 & $1,126,656$ & 43 & 35.1 & 1111 & 34 & $\begin{array}{l}1 \text { of } \\
\text { each }\end{array}$ & $\begin{array}{l}181 \\
(81.9 \%)\end{array}$ \\
\hline$w R i$ & Drosophila simulans & A & NC_012416 & $1,445,873$ & 1 & 35.2 & 1396 & 34 & $\begin{array}{l}1 \text { of } \\
\text { each }\end{array}$ & $\begin{array}{l}183 \\
(82.8 \%)\end{array}$ \\
\hline wSuz & Drosophila suzukii & A & $\begin{array}{l}\mathrm{NZ}- \\
\text { CAOU02000000 }\end{array}$ & $1,415,350$ & 110 & 35.7 & 1321 & 34 & $\begin{array}{l}1 \text { of } \\
\text { each }\end{array}$ & $\begin{array}{l}184 \\
(83.3 \%)\end{array}$ \\
\hline WVitA & Nasonia vitripennis & A & $\begin{array}{l}\text { NZ_- } \\
\text { MUJM01000000 }\end{array}$ & $1,211,929$ & 142 & 35.1 & 1097 & 34 & $\begin{array}{l}1 \text { of } \\
\text { each }\end{array}$ & $\begin{array}{l}185 \\
(83.7 \%)\end{array}$ \\
\hline wAlbB & A. albopictus & B & NZ_CP031221 & $1,484,007$ & 1 & 34.4 & 1418 & 34 & $\begin{array}{l}1 \text { of } \\
\text { each }\end{array}$ & $\begin{array}{l}180 \\
(81.4 \%)\end{array}$ \\
\hline$w C e r 5$ & $\begin{array}{l}\text { Rhagoletis cerasi } \\
\text { (RcerAS) }\end{array}$ & B & JADCNE01000000 & $1,180,723$ & 57 & 33.9 & 1091 & 34 & $\begin{array}{l}1 \text { of } \\
\text { each }\end{array}$ & $\begin{array}{l}179 \\
(81.0 \%)\end{array}$ \\
\hline$w D i$ & Diaphorina citri & B & СР051264 & $1,538,623$ & 1 & 33.9 & 1418 & 34 & $\begin{array}{l}1 \text { of } \\
\text { each }\end{array}$ & $\begin{array}{l}184 \\
(83.3 \%)\end{array}$ \\
\hline wNo & Drosophila simulans & B & NC_021084 & $1,301,823$ & 1 & 34.0 & 1220 & 34 & $\begin{array}{l}1 \text { of } \\
\text { each }\end{array}$ & $\begin{array}{l}184 \\
(83.3 \%)\end{array}$ \\
\hline wPip & Culex quinquefasciatus & B & NC_010981 & $1,482,455$ & 1 & 34.2 & 1410 & 34 & $\begin{array}{l}1 \text { of } \\
\text { each }\end{array}$ & $\begin{array}{l}181 \\
(81.9 \%)\end{array}$ \\
\hline wStri & Laodelphax striatellus & B & NZ_MUIX01000000 & $1,786,382$ & 2 & 33.8 & 1747 & 34 & $\begin{array}{l}1 \text { of } \\
\text { each }\end{array}$ & $\begin{array}{l}183 \\
(82.9 \%)\end{array}$ \\
\hline
\end{tabular}

differentiation of supergroup A and B genomes in terms of gene content (Additional File 1). The largest grouping included all the 19 genomes (738 orthogroups) but the next most abundant groups were exclusively the supergroup B strains (49 orthogroups) and supergroup A strains (28 orthogroups).

According to the maximum likelihood phylogenetic tree, $w$ Cer1 was basal to the clade containing $w$ Cer2 and $w$ Cer4. $w$ Cer4 was most closely related to $w$ Irr (Fig. 2), sharing 6 unique orthogroups. Prophage regions were identified using PHASTER, with four regions in wIrr which cumulatively equalled $73.3 \mathrm{~kb}$, while $w$ Cer 4 had two regions equalling $54.3 \mathrm{~kb}$ (Additional File 2). Similarly, $w$ Cer1 had two prophage regions equalling $45.1 \mathrm{~kb}$. It is possible that the fragmentation of the genome assemblies means that prophage regions that are split across contigs do not meet the threshold for identification. However, this was not supported by mapping of the reads from Ccap10.3 ( $w$ Cer 4$)$ and RcerHB $(w$ Cer 1$)$ onto $w \operatorname{Irr}$ at $90 \%$ similarity and $60 \%$ length, which showed that there were many genes in the prophage regions of $w$ Irr that were absent from $w$ Cer 4 


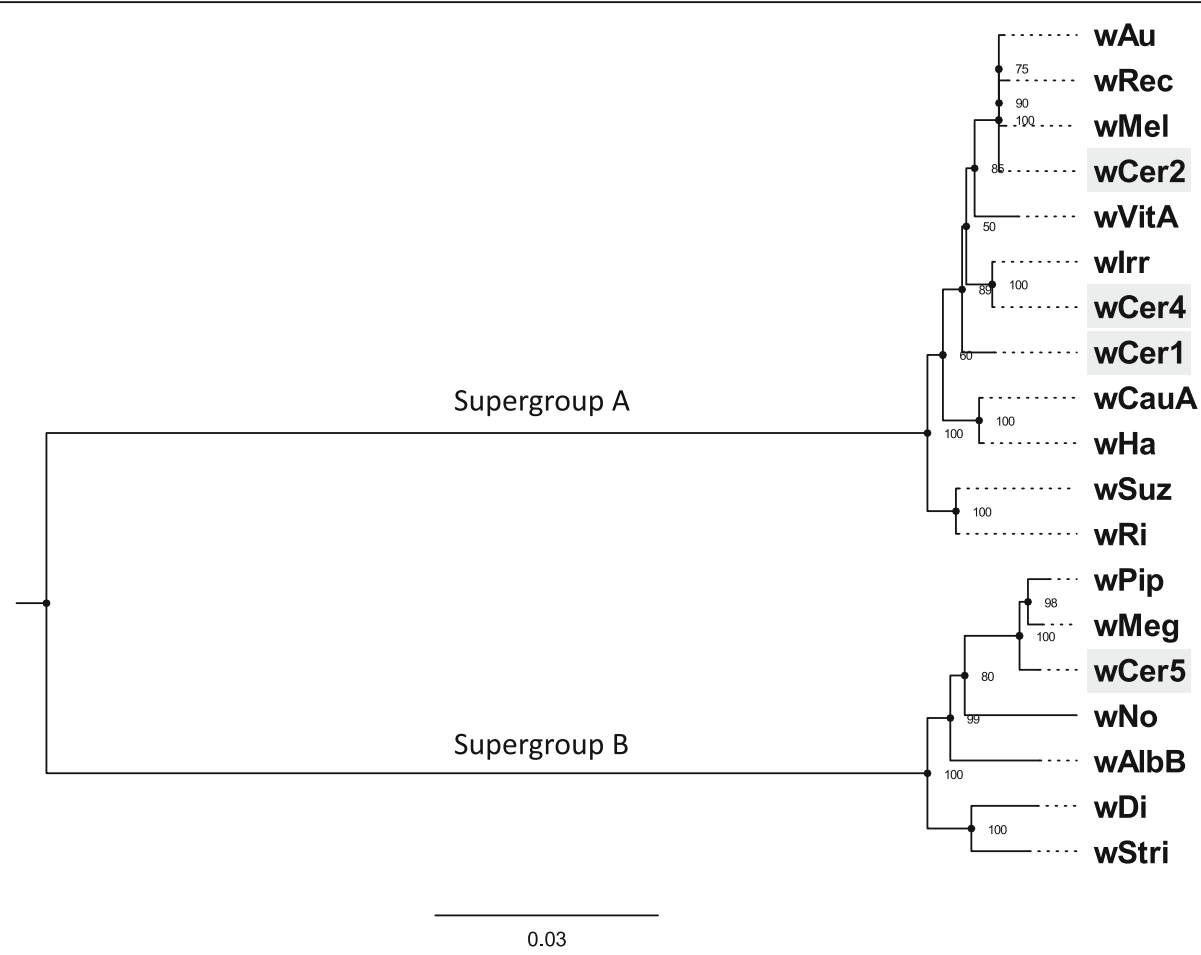

Fig. 2 Maximum likelihood tree of Wolbachia genomes based on 256 orthologous genes. These genes are single-copy coding genes present in each of the 19 genomes and include 183,819 nucleotide positions of which 25,996 are parsimoniously informative. The general time reversible base substitution model $(G T R+F+R 2)$ was used to produce the tree, supported by 1000 bootstrap repetitions. WCer genomes are shaded grey

and $w$ Cer1. In comparison, wCer2 had three annotated prophage regions, cumulatively equalling $170 \mathrm{~kb}$ [24], and, therefore, the largest prophage number and sequence length when compared to the other strains infecting this host species.

The $w$ Cer5 genome was most closely related to $w$ Pip and $w \mathrm{Meg}$, confirming its placement in supergroup B (Fig. 2), with seven orthologous groups unique to these three, six unique to $w$ Cer 5 and $w \mathrm{Meg}$, and three unique to $w$ Cer5 and $w$ Pip (Additional File 1). In comparison to the other strains $w$ Cer 5 had the smallest representation of prophage genes, with the presence of one incomplete region of $8.4 \mathrm{~kb}$ (Additional File 2).

\section{Cif and wmk genes in wCer genomes}

Orthology to verified cifA and cifB genes identified two pairs of Type I cif genes in $w C e r 1$; one pair of Type I plus one pair of Type V cif genes in wCer4; and one pair of Type IV cif genes in wCer5 (Table 3). Original annotation of $w$ Cer2 identified three pairs of Type I cif genes and a single Type V cifB gene [24], but reanalysis with OrthoFinder using six additional Wolbachia reference strains (particularly $w$ StriCN and $w$ Irr) improved the identification of Type $\mathrm{V}$ cif genes, and the hypothetical gene E3V96_3725 contiguous with the previously identified Type $\mathrm{V}$ cifB was annotated as $c i f A_{w C e r 2[T 5] .}$ Therefore, $w$ Cer2 had four complete sets of cif genes and the largest number of cif modules in this host species.

The cifA maximum likelihood tree comprised 41 cifA orthologues, representative of all five types, and was built on an alignment of 1884 nucleotide sites, of which 1267 were parsimony-informative (Fig. 3; Additional File 3). The cifB gene alignment comprised 39 genes representative of all five types, with 5093 nucleotide sites of which 2651 were parsimony-informative (Fig. 4; Additional File 3).

Both contiguous cif gene pairs in $w$ Cer1 were similar to the $c i f_{w P i p[T 1]}$ archetypes and contained complete functional domains and conserved amino acids of the Type I cif genes (Fig. 5). wCer4 also contained complete and potentially functional $c i f_{w P i p[T 1]}$-like genes, in addition to $c i f_{w C e r 4[T 5]}$ genes where ${ } i f B_{w C e r 4[T 5]}$ had a 2511 amino acid extension of ankyrin and latrotoxin domains and furin cleavage sites. This was similar to other Type $\mathrm{V}$ cifB genes including the $\operatorname{cif} B_{w \operatorname{Cer} 2[T 5]}$ gene E3V96_03720 [24], which also had an ankyrin and latrotoxin extension (Fig. 5). Both of these genes were similar in the PDDEXK nuclease domains to the Type IV and Type $\mathrm{V}$ cifB genes of $w$ Pip and $w$ StriCN respectively, 
Table 3 cif genes in Wolbachia strains of Rhagoletis cerasi

\begin{tabular}{|c|c|c|c|c|c|c|}
\hline & Gene & Size (aa) & Locus & $\begin{array}{l}\text { Orthologue, size (aa), \% } \\
\text { similarity }\end{array}$ & Type & Evidence for $\mathrm{Cl}$ \\
\hline \multirow[t]{4}{*}{ wCer1 } & INQ25_05555 & 491 & cifA & wHa_RS01435, 492, 91\% & I & \multirow{4}{*}{$\begin{array}{l}\text { UNCERTAIN: No crossing experiment data exists; but strong } \\
\text { linkage with mitochondrial haplotype (HT1) in Rhagoletis cerasi } \\
\text { field populations }\end{array}$} \\
\hline & INQ25_05550 & 1143 & cifB & wHa_RS01430, 1148, 91\% & 1 & \\
\hline & INQ25_01115 & 491 & cifA & wHa_RS01435, 492,88\% & । & \\
\hline & INQ25_01120 & 1150 & cifB & wHa_RS01430, 1148, 92\% & 1 & \\
\hline \multirow[t]{8}{*}{$w C e r 2$} & E3V96_03425 & 475 & cifA & WMel_RS02835, 475, 99\% & । & \multirow{8}{*}{$\begin{array}{l}\text { YES: Experimental evidence for } \mathrm{Cl} \text { in multiple novel hosts - } \\
\text { Drosophila simulans, Ceratitis capitata, Bactrocera oleae; } \\
\text { strong linkage of wCer2 with mitochondrial haplotype (HT2) } \\
\text { in R. cerasi field populations }\end{array}$} \\
\hline & E3V96_03430 & 1174 & cifB & WMel_RS06940, 1174, 99.7\% & । & \\
\hline & E3V96_02935 & 481 & cifA & WVitA_RS00555, 499, 75\% & । & \\
\hline & E3V96_02940 & 1531 & cifB & WVitA_RS00550, 1523, 85\% & । & \\
\hline & E3V96_06520 & 492 & cifA & wPip_RS01410, 504, 90\% & । & \\
\hline & E3V96_06515 & 921 & cifB & wPip_RS01415, 1175, 83\% & । & \\
\hline & E3V96_03725 & 438 & cifA & $\begin{array}{l}\text { Wirr_E0495_RS03300, 429, } \\
66 \%\end{array}$ & V & \\
\hline & E3V96_03720 & 3405 & $c i f B$ & $\begin{array}{l}\text { wStriCN_BVG17_RS00730, } \\
3083,72 \%\end{array}$ & V & \\
\hline \multirow[t]{4}{*}{ wCer4 } & INQ27_01280 & 494 & cifA & wHa_RS01435, 492, 79\% & । & \multirow{4}{*}{$\begin{array}{l}\text { YES: Experimental evidence for strong } \mathrm{Cl} \text { in novel host } \\
\text { C. capitata }\end{array}$} \\
\hline & INQ27_01275 & 1166 & cifB & wPip_RS01415, 1175, 85\% & । & \\
\hline & INQ27_01270 & 415 & cifA & $\begin{array}{l}\text { wStriCN_BVG17_RS06595, } \\
415,84 \%\end{array}$ & V & \\
\hline & INQ27_01265 & 3332 & cifB & $\begin{array}{l}\text { WStriCN_BVG17_RS06590, } \\
4358,72 \%\end{array}$ & V & \\
\hline \multirow[t]{2}{*}{ wCer5 } & INQ21_01080 & 446 & cifA & wPip_RS01460, 446, 99.8\% & IV & \multirow{2}{*}{$\begin{array}{l}\text { UNCERTAIN: No crossing experiment data exists; but very high } \\
\text { similarity of cif gene sequences to the Type IV cifA/B genes of } \\
\text { wPip with proven } \mathrm{Cl} \text { induction }\end{array}$} \\
\hline & INQ21_01085 & 733 & $c i f B$ & wPip_RS01465, 733, 99.6\% & IV & \\
\hline
\end{tabular}

notably to the conserved amino acids identified by Shropshire et al. [54]. wCer5 is a supergroup B strain most closely related to $w$ Pip and $w$ Meg, containing a single contiguous pair of $\mathrm{cif}_{w \operatorname{Cer} 5 \text { [T4] }}$ genes that shared 99.8\% (cifA) and 99.6\% (cifB) amino acid similarity with CI inducing cif $_{\text {wPip [T4]. }}$

Furthermore, we found a wCer2 gene (E3V96_03405) with an identical amino acid sequence to $w m k$ of $w \mathrm{Mel}$ (WD0626) which had previously been found to cause MK when highly expressed in transgenic D. melanogaster. No orthologues for this gene were found in $w$ Cer4 and $w$ Cer5. However, for a wmk homologue in $w \mathrm{Mel}$, WD0508, for which transgenic expression did not alter sex ratios in D. melanogaster, orthologues were found in $w$ Cer2 (similarity of $89 \%$ ), wCer4 (94\%) and $w$ Cer5 (93.6\%). No orthologues of $w m k$ or its homologues were found in $w$ Cer1.

\section{Mitochondrial genome polymorphisms in different populations}

The mitochondrial genomes assembled from the three $R$. cerasi individuals were representatives of three (including geographically distant and genetically diverged) populations with different Wolbachia infection types
(Additional File 4). Mitogenome comparisons revealed 17 SNPs and three indels in homopolymer regions between RcerHB and RcerIZ; 24 SNPs and five indels between RcerHB and RcerAS; and 29 SNPs and four indels between RcerIZ and RcerAS (Fig. 6). The two haplotypes HT1 and HT2 previously defined by one SNP difference (SNP position 2767 of the mitogenome) were found to be linked with $w$ Cer1 and $w$ Cer2, respectively. More specifically, the mitogenomes of the individuals RcerHB and RcerIZ were HT1 (denoted HT1a and $\mathrm{HT} 1 \mathrm{~b}$, respectively) and these individuals did not have $w$ Cer2, and the mitogenome of $w$ Cer2-infected RcerAS was HT2 (Fig. 1).

A population genomic comparison of the level of mitogenome divergence within the two mitochondrial haplotypes was not possible due to the low sample replication of two HT1 mitogenomes and one HT2 mitogenome. However, mitochondrial haplotype differences were analysed and linked to $w$ Cer2 presence or absence using a previously published ddRadseq dataset, which included 192 R. cerasi individuals from different locations with different Wolbachia strain combinations. Of the 41 differing sites (either SNPs or indels) between the three mitogenomes (Additional File 4), only 12 were 


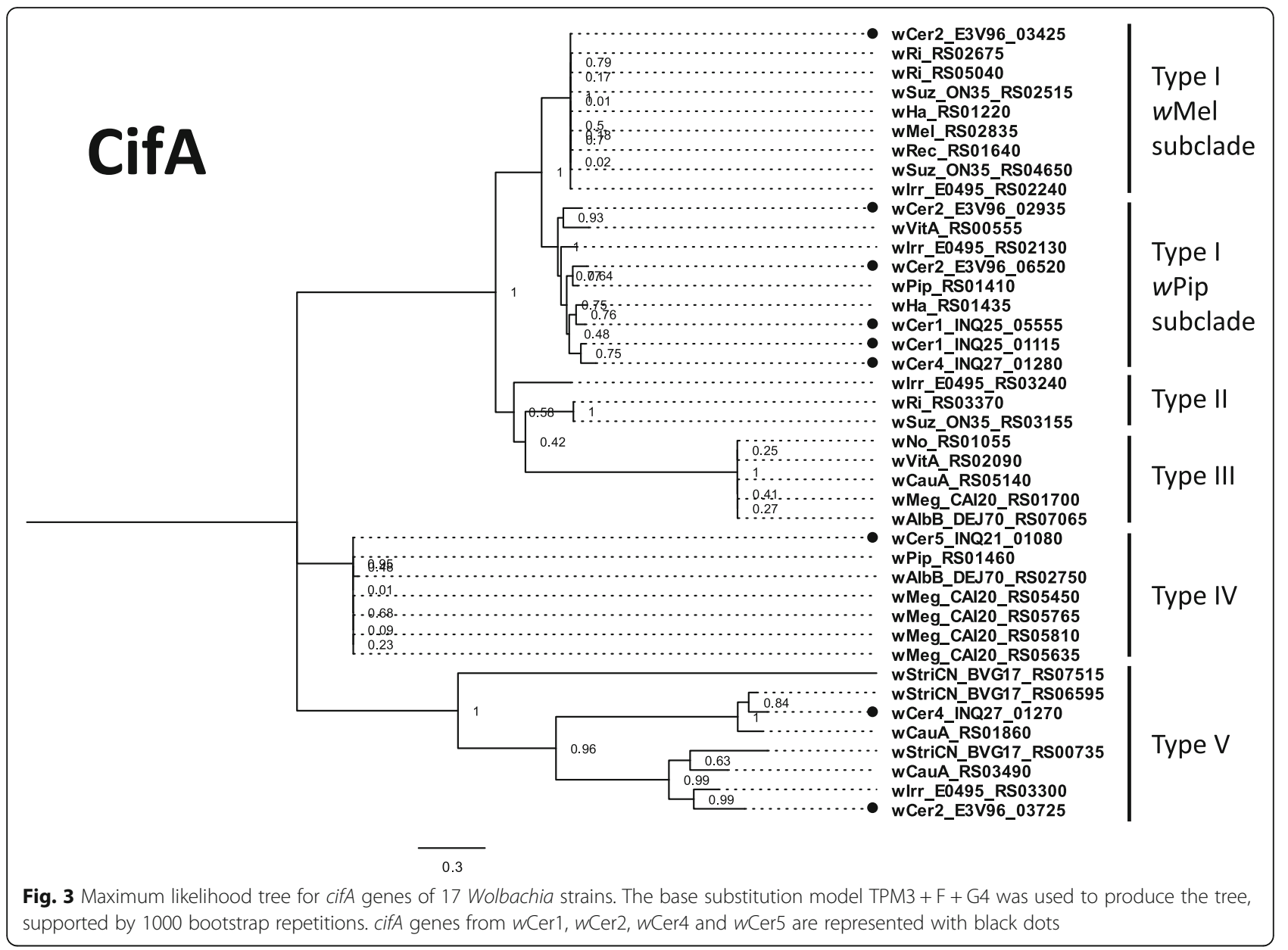

represented by ddRadseq loci (Additional File 5). For HT1 individuals (without $w$ Cer2), 8 of the 12 sites were polymorphic (32 individuals including 30 ddRadseq individuals plus RcerHB and RcerIZ); for HT2 individuals (with $w$ Cer2) only 10 sites were represented by ddRADseq, and none was polymorphic (16 individuals including 15 ddRadseq individuals plus RcerAS). The variance in the Euclidean distance within the HT1 group of mitochondrial haplotypes $(\mathrm{var}=0.07)$ was higher than group HT2 (var=0.001). Furthermore, the PERMANOVA showed strong influence of the $w$ Cer1/wCer2 grouping on the distance measures between haplotypes $(p=0.001)$, but presence of $w$ Cer4 $(p=0.736)$ or $w$ Cer5 $(p=0.206)$ had no effect (Additional File 6).

\section{Wolbachia strain polymorphisms across populations}

The variation across the Wolbachia strains independently isolated from geographically distant and genetically diverged host populations was investigated to infer the relative ages of the associations of $w$ Cer1, $w$ Cer 2 and $w$ Cer5 with $R$. cerasi. Consensus sequences (Fig. 6;
Additional Files 7, 8 and 9) and variant information (Additional Files 10, 11 and 12) were extracted for each combination of strain and host population. Genome networks of complete consensus sequences for each strain, $w$ Cer1 (RcerAS, RcerIZ, RcerHB), wCer5 (RcerAS, RcerIZ) and $w$ Cer2 (RcerAS, and three microinjected novel hosts $C$. capitata Ccap88.6, D. simulans DsimRC45, DsimRC50 [24]) showed that the numbers of SNPs between variants of strains across populations varied (Fig. 6), but no rearrangements or large gaps in sequence were noted. The number of SNPs in consensus sequences between the two $w$ Cer5 variants (RcerAS, RcerIZ; 29 SNPs) was almost 60\% higher than the number of SNPs between the two $w$ Cer1 variants in the same two samples (RcerAS, RcerIZ; 19 SNPs). The consensus sequences for each strain for each individual were calculated by majority consensus with no lower coverage threshold, however the additional variant analysis also showed strain variation within host individuals. The $w$ Cer1 of RcerHB showed at least two possible nucleotides (with minimum coverage of $35 \%$ of a given variant with minimum coverage threshold of 5 reads) at 16 sites; 


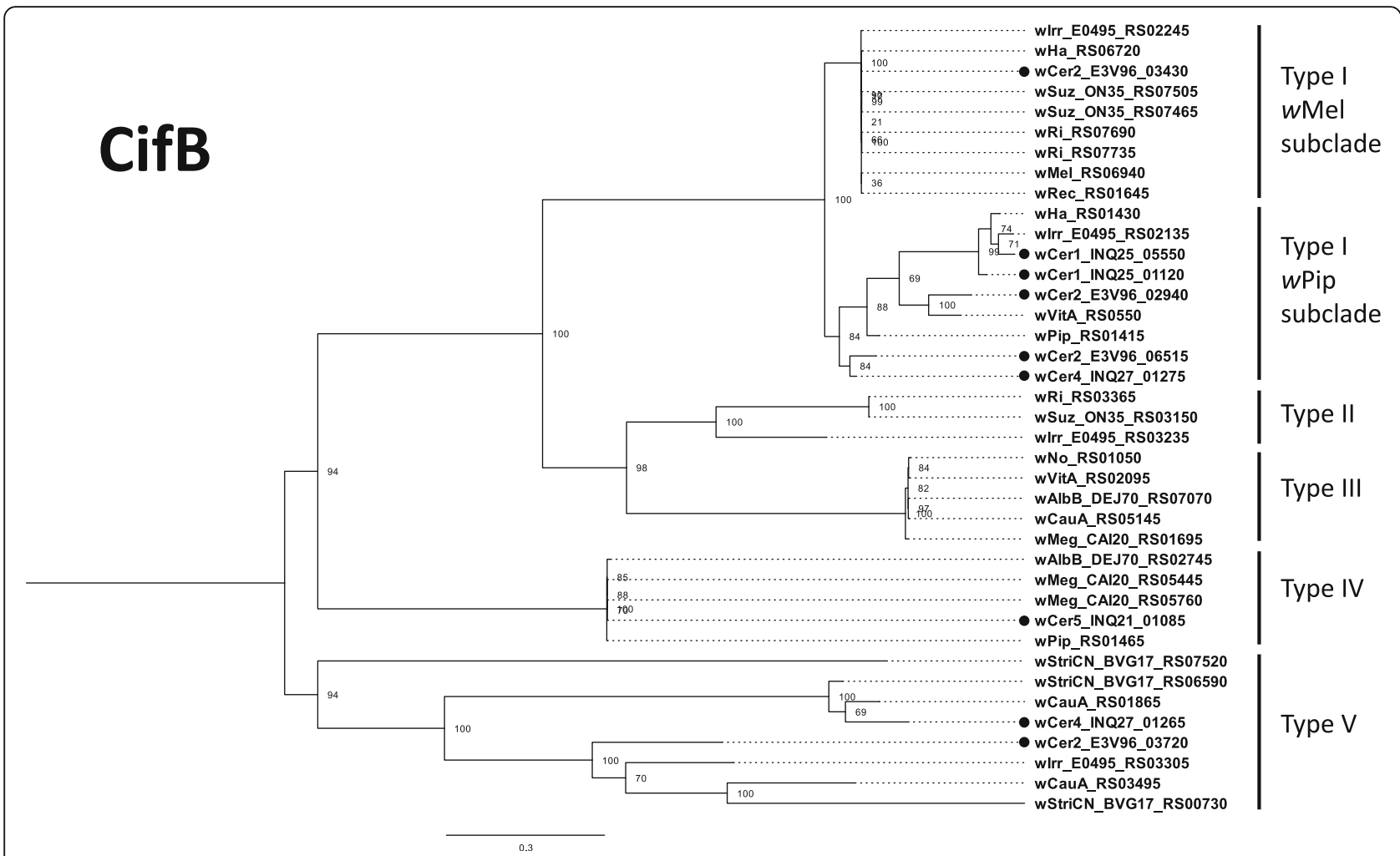

Fig. 4 Maximum likelihood tree for cifB genes of 17 Wolbachia strains. The base substitution model TPM $3+F+I+G 4$ was used to produce the tree, supported by 1000 bootstrap repetitions. cifB genes from wCer1, wCer2, wCer 4 and wCer 5 are represented with black dots

at 12 of these sites both nucleotides were present in the $w$ Cer1 of RcerIZ, and at 10 sites both nucleotides were present in the $w$ Cer1 of RcerAS. The overlap of variant sites in RcerIZ and RcerAS was at 11 sites (Additional File 10). The $w$ Cer5 strain variation within each individual accounted for only four of the 29 SNPs between the $w$ Cer5 variants of RcerAS and RcerIZ (Additional File 11 ). To ensure these results were not inflated by the collapse of non-identical multicopy genes into single genes in the draft genome, the sequence locations of the SNPs were determined (Additional Files 10 and 11). SNPs were found mostly in intergenic regions, single copy genes or multiple copy genes where $w$ Cer 1 or $w$ Cer 5 had similar orthologues to the reference genomes (based on $w$ Cer1, $w \mathrm{Au}$ and $w \mathrm{Mel}$ genomes for $w$ Cer1 SNPs; and $w$ Cer5, $w$ Meg and $w$ Pip genomes for $w$ Cer5 SNPs). However, our data indicated at most a single SNP in a transposase gene that was single copy in $w$ Cer1 but multicopy in $w \mathrm{Au}$ and $w \mathrm{Mel}$ may be a false positive. For $w$ Cer5, the single SNP in the phage gene patatin and nine of the 10 SNPs in the major tail sheath protein gene in RcerAS are true differences (> 58\% frequency) from the reference sequence, while one heterozygous site may be a false positive. Notwithstanding these possible misassemblies, the number of changes still suggests $w$ Cer5 has a greater number of SNPs than $w$ Cer1 between RcerIZ and RcerAS.

For wCer2, eight SNPs between the Wolbachia genome of Ccap88.6 and its donor RcerAS (Fig. 6) were detected; six or seven SNPs differentiated $w$ Cer2 variants in two D. simulans hosts (DsimRC50 and DsimRC45) from their donor, but three SNPs were common to the two recipients, and an additional two were common to the Ccap88.6 and D. simulans lines (Fig. 6). All variants except one (for which $100 \%$ reads were different from the reference nucleotide) were found in the $w$ Cer2 of RcerAS.

We also searched the ddRadseq reads mapped to the Wolbachia strains to find SNPs that would extend our dataset, and used the mapped reads to establish the infection status of each individual, but very few reads mapped to sites previously identified as polymorphic, and we were unable to extend analysis of Wolbachia strain genomic variation across more populations than those that were whole genome sequenced. However, the relative titre of the strains in each of the 46 ddRADseq samples which were informative was assessed using the mapping coverage of the ddRADseq reads on each $\mathrm{Wol}$ bachia strain at a minimum of five sites. Consistently $w$ Cer2 had high coverage $(>30 \mathrm{x}), w$ Cer1 had $\sim 20 \mathrm{x}$ 


\section{CIFA and CIFB contiguous pairs}
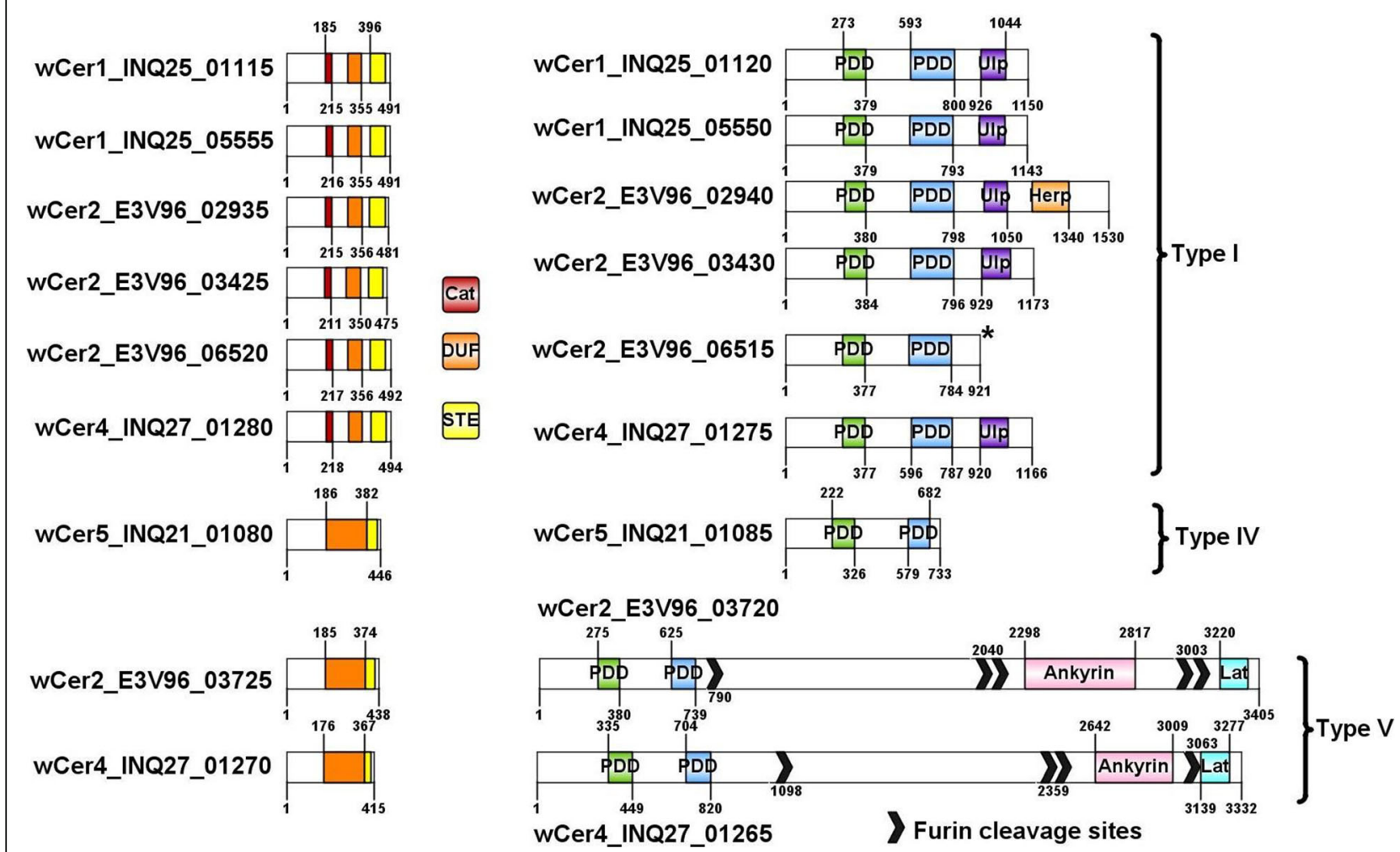

Fig. 5 Protein domain structure of CifA and CifB of wCer1, wCer2, wCer4 and wCer5

coverage, $w$ Cer 4 had $\sim 2 \mathrm{x}$ coverage and $w$ Cer 5 had $\sim 5 \mathrm{x}$ coverage, thereby confirming $w$ Cer 1 and $w C e r 2$ as high titre infections, and $w$ Cer 4 and $w$ Cer 5 as low titre infections in this species.

The number of mutations between the $w$ Cer1 variants and between the HT1 mitogenomes of RcerIZ and RcerHB (11 SNPs and 17 SNPs respectively), equated to $117 \mathrm{x}$ relatively more mutations in the mitogenome (Wolbachia genome is approximately 76x larger than the mitogenome). The $w$ Cer1 genome comparison of RcerAS and RcerHB revealed 14 SNPs, the mitogenomes of those samples had 24 SNPs, which equated to 130x relatively more mutations in the mitogenome. The $w$ Cer1 genome comparison of RcerAS and RcerIZ revealed 19 SNPs, the mitogenomes of those samples differed by 29 SNPs, i.e. $116 x$ relatively more mutations in the mitogenome.

\section{Discussion}

We sequenced and assembled three new Wolbachia strain genomes, $w$ Cer1, $w$ Cer 4 and $w C e r 5$, of the European cherry fruit fly, R. cerasi, and analysed these in conjunction with the previously sequenced genome of $w$ Cer2 [24]. All genomes contained cif genes with functional domains which indicates $\mathrm{CI}$ is likely induced by all four strains. The larger number and diversity of prophage-associated cif gene modules, and lower Wolbachia strain and mitochondrial genome variant diversity associated with $w$ Cer2 support the hypothesis that $w$ Cer2 is the most recently acquired Wolbachia strain in this host species, while $w$ Cer 1 has been associated with $R$. cerasi for a longer period. The higher level of polymorphism between $w$ Cer 5 variants than between wCer1 variants in individuals of geographically distant populations suggests an even longer association of $w$ Cer 5 with $R$. cerasi. While the finding of cif genes with functional domains in $w$ Cer1 together with its fixation in all $R$. cerasi populations are indicators of strong CI expression by this strain in this host species, this is less clear for $w$ Cer 4 and $w$ Cer 5 because of their patchier geographic distribution and more moderate prevalence in host populations when compared to $w$ Cer 1 and $w$ Cer2 [14], without any clear linkage of $w$ Cer4 and $w$ Cer 5 to mitochondrial haplotypes. However, $w$ Cer4 causes strong CI in C. capitata [42], and $w$ Cer5 is fixed in some populations of $R$. cerasi [14]. The loss of any linkage of $w$ Cer 4 and $w$ Cer 5 with a 


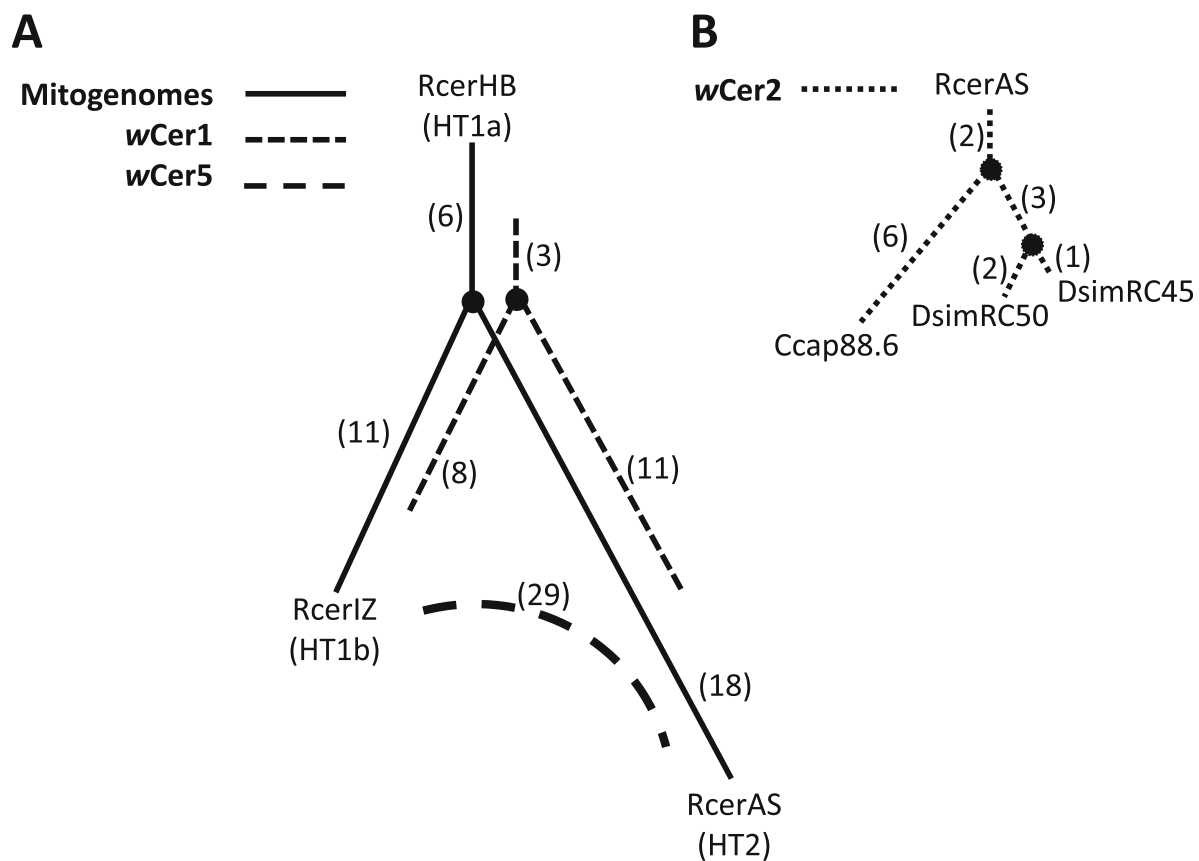

Fig. 6 Haplotype networks for (A) mitogenome variants of RcerHB (HT1a), RcerlZ (HT1a) and RcerAS (HT2) and Wolbachia strains wCer1, wCer2 and wCer5, and (B) wCer2 variants between RcerAS and three novel host lineages (Ccap88.6, DsimRC45 and DsimRC50) infected by embryonic microinjection. Numbers (in parentheses) next to solid lines indicate SNPs between the mitogenome variants, and numbers (in parentheses) next to broken lines SNPs between the Wolbachia strain genome variants. Input alignments were based on library-specific consensus genomes (Additional Files 7, 8 and 9) determined by majority rule (nucleotide called when $>50 \%$ of mapped reads). wCer 4 was not included in this analysis because only one genome variant was sequenced

particular mitochondrial haplotype could indicate that these two strains colonised $R$. cerasi prior to the invasion by $w$ Cer1 and $w$ Cer2, and their lower prevalence and/or patchier distribution may indicate that their CI phenotype may be (i) weak (due to their lower titre), (ii) partially supressed by $R$. cerasi (as seen for $w$ Mel in Drosophila melanogaster $[55,56])$, or alternatively, (iii) $w$ Cer1 and/or $w$ Cer2 may be able to rescue some of the CI induced by $w$ Cer4. The latter is unlikely for $w$ Cer 5 , because its Type IV cif gene pair is unique in this host system. Furthermore, while the genome analyses revealed high similarities to the $w m k$ genes of $w \mathrm{Mel}$ and $w \operatorname{Rec}$ in $w$ Cer2, it is unlikely they cause MK in this host system, also because sex ratio biases have so far not been reported. Future research should investigate whether $w C e r 4$ and $w C e r 5$ are indeed maintained in populations by $\mathrm{CI}$, and if so, how their variable prevalences found across populations affect the ongoing invasion of $R$. cerasi by $w$ Cer2. Alternatively they are maintained because of beneficial host effects as seen for non-CI or weak CI-inducing strains in some host species [55, 57, 58]. An increased knowledge base surrounding the interactions of multiple CI-inducing Wolbachia strains in the same host species (including after artificial introduction by microinjection) is crucially important in Wolbachia-based management of pest and vector populations which are already infected by Wolbachia (e.g. $[59,60])$.

\section{Cif and wmk genes in multiple co-occurring Wolbachia strains}

When comparing the cif gene repertoires of the Wolbachia strains, wCer1 has two intact pairs of cif genes, similar to the $c i f_{w P i p[T 1]}$ genes that recapitulate CI [21]; $w$ Cer2 has two Type I cif gene pairs also in the $w$ Pip Type I sub-clade with an additional Type I cif gene pair, almost identical to the archetypal $c i f_{w M e l[T 1]}$ gene pair $[20,24]$, and a $c i f_{w C e r 2[T 5]}$ gene pair for which only cifB had previously been annotated [24]. All Cif proteins in $w$ Cer1 (two pairs) and $w$ Cer2 (four pairs) appear to have necessary functional domains, so, while there is no evidence from crossing experiments, $w$ Cer2 may have the potential to rescue $w$ Cer1-induced CI. This is most likely via the two closely related cifA from the cif $_{\text {wPip[T1] }}$ subclade, however the $w$ Mel-like Type I cifA gene could also play a role. Such a prediction is supported by the demonstrated CifA rescue of $\mathrm{CI}$ induced by a similar but non-cognate CifA/B pair [28]. Conversely, the presence of multiple cif genes of the same type may cause additional CI and/or strengthen CI levels. 
Furthermore, cif $f_{w C e r 2[T 5]}$ may induce CI that is not rescued by Type I cif due to their dissimilarity. While there is no experimental evidence for $\mathrm{CI}$ induction by $c i f_{w-}$ Cer2[T5], Type V cif genes have characteristics of other cif types: (i) the cifA and cifB genes are adjacent, transcribed in the same direction and located in prophage regions; (ii) the domain structure is similar to cif $_{\text {wPip [T4] }}$ (Fig. 5), which has been determined to recapitulate CI in a transgenic system [21]; and (iii) potential functionality of Type $\mathrm{V}$ cif genes is demonstrated in the CI-inducing strain wStri which only contains Type V cif genes [26, 27]. However, to unequivocally discern the contribution to CI of individual cif pairs expressed in a Wolbachia strain containing multiple copies, transgenic expression of single cif genes or gene pairs is necessary.

Furthermore, wCer4 induces CI and is bidirectionally incompatible with $w$ Cer2 in the novel host $C$. capitata [42]. We found that $w$ Cer4 had one pair of Type I cif genes encoding intact functional domains and were phylogenetically grouped in the cif $B_{w P i p[T 1]}$ subclade. $w$ Cer4 also had a Type V cif gene pair which was most similar to cif $_{\text {wStriCN[T5] }}$ (84 and $72 \%$ amino acid similarity respectively) but only had 36 and $45 \%$ similarity with

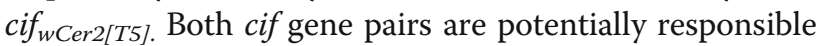
for $\mathrm{CI}$ in $w$ Cer4 and one or both are presumably incompatible with the $w$ Cer 2 cif gene pairs. The substantial divergence between the Type $\mathrm{V}$ cif genes in $w$ Cer2 and $w$ Cer4 suggests that they are likely incompatible. The repertoire of cif genes in $w$ Cer 4 also indicates this strain may be incompatible with $w$ Cer1, due to the absence of Type $\mathrm{V}$ cif genes in $w$ Cer 1 .

While it has not been demonstrated in crossing experiments that $w$ Cer5 can induce $\mathrm{CI}$, the genome sequencing of $w$ Cer5 showed a high similarity of $c i f_{w C e r 5[T 4]}$ to CI-inducing cif $f_{\text {wPip [T4] }}$ (over 99.6\% amino acid identity), and is therefore likely to induce CI. Furthermore, $w$ Cer5 is the only $w$ Cer genome with Type IV cif genes in this host species, and unlike the other $w$ Cer genomes has no Type I cif genes. Therefore, $w$ Cer5 is likely bidirectionally incompatible with $w$ Cer $1, w$ Cer 2 and $w$ Cer 4 , and this could cause issues, e.g. slowing down of a $w$ Cer 2 invasion between populations that are polymorphic in infection status. One would expect that for invasion to be successful either $w$ Cer2 and $w$ Cer5 spread should be linked, or $w$ Cer5 should already exist at a high enough prevalence in populations that are being invaded.

We have also investigated whether the $w$ Cer genomes contain orthologues of the wmk gene sequence which can simulate a MK phenotype in transgenic D. melanogaster [50]. While $w$ Cer2 has a gene with identical amino acid sequence, the other strains only have similarities to a wmk homologue which does not simulate MK. Furthermore, no sex ratio distortion has previously been observed in $R$. cerasi and novel hosts of $w$ Cer2 and $w$ Cer 4 .
Therefore, it is unlikely MK is expressed in this host system.

\section{Incomplete self-rescue or fitness costs of $w$ Cer 2 and $w$ Cer4 in novel hosts}

An unresolved aspect to the phenotype of $w$ Cer2 and $w$ Cer4 in novel hosts D. simulans [41], C. capitata [42] and B. oleae [43] involves the reduced hatch rate seen in crosses involving parents that are infected with the same strain. This phenotype may be attributed to a fitness cost independent of $\mathrm{CI}$, such as is associated with high Wolbachia titre [61], or incomplete self-rescue because uninfected control crosses and crosses between uninfected males and $w$ Cer2 or $w$ Cer4 infected females had significantly lower embryonic mortality.

We previously annotated a single unpaired Type V cifB gene in $w$ Cer2, which we hypothesised either (i) caused lethality by toxic expression; (ii) induced weak $\mathrm{CI}$ when combined with a non-cognate cifA gene; or (iii) induced $\mathrm{CI}$ that was only partially rescued through activity of a non-cognate cifA gene [24]. However, reanalyses found that this $c i f B$ gene was not unpaired, and its relatively more diverged cifA partner has now been annotated due to the addition of more diverse cif gene types in the comparative genome analysis. Similarly, we also identified additional Type $\mathrm{V}$ cifA genes in wIrr [25] and $w$ CauA which also exemplify the substantial diversity within the Type $\mathrm{V}$ clade.

While unpaired $c i f B$ genes are not involved in incomplete self-rescue, toxicity has been demonstrated in transgenic insects for $\operatorname{cif} B_{w \operatorname{Rec}[T 1]}$ [28] and $c i f B_{w-}$ Pip [T4] [62] when the cifB transgene is expressed alone in males. Furthermore, cifB $B_{w R e c[T 1]}$ cannot be rescued by either cognate cifA or non-cognate $c i f_{w M e l[T 1]}$, so lethality is not strictly through CI [28], and this effect may only be indirectly related to CI competence. Incomplete self-rescue has not been reported for either Wolbachia strains $w \operatorname{Rec}$ or $w$ Pip, so the previously mentioned results were obtained through sole transgenic expression of cifB. However, different expression levels in a natural situation may emulate this outcome. Therefore, expression studies that compare individual transcription levels of the cif gene repertoire of $w$ Cer 2 and $w$ Cer 4 may resolve this question. Similar research should also be done with $w$ Tei, another strain that experiences incomplete self-rescue upon its transfer from its original host Drosophila teissieri to D. simulans [63].

wCer1 is fixed in $R$. cerasi, but wCer 5 has a longer host association

Based on consensus sequence comparisons, the divergence between $w$ Cer5 variants is larger than the 
divergence between the respective $w$ Cer1 variants (by $60 \%)$. This indicates that $w$ Cer 5 has been associated with $R$. cerasi for a relatively longer period than $w$ Cer1. Furthermore, the variant analysis showed that strain variants comprised a polymorphic population. For $w$ Cer5 there were few variable site overlaps, and therefore more accrued (or real) differences between the two sequenced individuals; in contrast, there were many variable site overlaps for $w$ Cer 1 variants of the same two individuals. The linkage of $w$ Cer1 with HT1 is a clear indication of a more recent invasion of $w$ Cer1 than $w$ Cer5. No such link for $w$ Cer5 with a mitochondrial haplotype was detected, and this could indicate that a previously existing linkage may have broken down as a consequence of the $w$ Cer1 invasion in $R$. cerasi. $w$ Cer5 maintains high prevalence in some $R$. cerasi populations where it routinely co-occurs with $w$ Cer1, $w$ Cer2 and $w$ Cer4 (Additional File 5), but is low or absent in other populations [14]. It was consistently detected at low coverage in the WGS reads and ddRADseq suggesting low titre in this host. Its patchier distribution, however, suggests it did not invade all populations, or has been lost from some populations, which may be due to incomplete transmission or high fitness costs. Low titre infections may reduce transmission success [64], or cause weak or ineffective CI [65, 66]. Low titre of strains may be attributed to competition with other $w$ Cer strains, diapause effects $[16,67]$, male age or male development time $[55,56]$, but evidence for this will be required from experiments that directly test transmission, $\mathrm{CI}$ and fitness costs of $w$ Cer5.

\section{Effects of wCer2 invasion on mitogenome and Wolbachia variant diversity}

It has been demonstrated that $w$ Cer2 is currently invading $R$. cerasi populations $[13,46]$, and this has caused a mitochondrial selective sweep [37, 47], previously only characterised by a single nucleotide difference in the COI gene [45]. We have revealed more mitogenomic variation between the genome sequenced individuals and extended the analysis using ddRADseq reads of 46 individuals from a large geographic area. We did not find any detectable mitogenomic variation in any of the 10 informative sites of individuals with $w$ Cer2, whereas mitogenomic variation was detected in 8 of the 12 informative sites across individuals with HT1 haplotypes and without $w$ Cer2. While mitochondrial network analysis could not be rooted with uninfected haplotypes because $w$ Cer 1 is fixed in this species, our data showed two-thirds of the variability between HT1 and HT2 haplotypes was found within the HT1 mitogenomes. This variability at multiple nucleotide sites within HT1 supports the previously detected mitochondrial hitchhiking due to the $w$ Cer2 invasion [37], and suggests that HT1 variability has been acquired by $R$. cerasi since invasion by $w$ Cer1.

Besides the mitochondrial sweep caused by a CIdriven invasion of a Wolbachia strain, it is also expected that any co-infecting strain already resident in host populations will also experience a selective sweep. For $R$. cerasi, such cytoplasmic hitchhiking is expected to be seen for $w$ Cer1, $w$ Cer 4 and $w$ Cer 5 associated with the invading $w$ Cer2 strain, and will result in loss of accumulated intrastrain diversity across populations, whereas $w$ Cer1, $w$ Cer 4 and $w$ Cer5 not associated with $w$ Cer2 will maintain any original intrastrain diversity. While we found SNPs within $w$ Cer strain genomes, due to insufficient coverage from the ddRadseq dataset, no additional information could be extracted to test for cytoplasmic hitchhiking of $w$ Cer1, wCer 4 and $w$ Cer 5 . However, the newly established genome data provide the basis for such investigations in the future.

Previously it was thought, based on identical MLST profiles of $w$ Cer2 and $w$ Cin 2 of the North American eastern cherry fruit fly Rhagoletis cingulata which has more recently become invasive in Europe, that $w$ Cer2 may have been acquired by $R$. cerasi from $R$. cingulata. However, this was disproven as, besides the MLST genes, the genomes of $w$ Cer 2 and $w$ Cin 2 are fairly distinct from each other [44]. However, while the origin of $w$ Cer2 in $R$. cerasi is still unclear, our mitogenome diversity analyses still suggest a fairly recent introduction of $w$ Cer2 to $R$. cerasi. In contrast, the high variation in mitochondrial haplotypes associated with $w$ Cer1 suggests a longer association with $R$. cerasi. The evidence previously presented that $w$ Cer1 is the source of the horizontal acquisition of $w$ Cin 1 in $R$. cingulata as part of its colonisation of Europe [68] will still need further validation by sequence analysis of the $w$ Cin 1 genome and comparison with the $w$ Cerl genome presented here.

\section{Conclusions}

Our analyses of the four Wolbachia genomes wCer1, $w$ Cer2, $w$ Cer 4 and $w$ Cer5 have provided insights into the diversity and modularity of cif gene interactions in the multiply infected host species $R$. cerasi. Next, more directed studies should be performed to investigate the capacity of each cif gene module in $w$ Cer genomes to induce and rescue $\mathrm{CI}$, understand the interaction of multiple cif gene modules when expressed in the same strain (such as for $w$ Cer1, $w$ Cer2 and $w$ Cer 4 ) or multiple strains in a single host, and resolve the fitness costs (toxicity or incomplete CI self-rescue) that have been demonstrated for single infections of $w$ Cer2 and $w$ Cer 4 in novel hosts. Furthermore, mitogenomes and Wolbachia genomes from WGS projects can guide and increase resolution of SNP analyses from reduced representation 
genomic datasets, such as ddRadSeq. This enabled us to link Wolbachia strain infection with mitogenome haplotypes in individuals and clearly demonstrated haplotype variation associated with Wolbachia infections and the more recent acquisition of $w$ Cer2.

\section{Methods}

\section{Source of individuals for genome sequencing}

One individual was sourced for genome sequencing from each of three populations of $R$. cerasi: RcerAS from Stillfried, Austria (approximately $40 \mathrm{~km} \mathrm{NE}$ of Vienna), collected in 2001; RcerIZ from Zafferana in eastern Sicily, Italy, collected in 2001; and RcerHB from Bajna, Hungary (approximately $40 \mathrm{~km} \mathrm{NW}$ of Budapest), collected in 2000 (Fig. 1). The geographic distances between sites ranged from 200 to $2000 \mathrm{~km}$, with a high host genetic divergence of $R$. cerasi between Sicily and the two central European sites [53]. Based on Wolbachia strain-specific PCR, individuals from Stillfried carried both $w$ Cer1 and $w$ Cer2 while the individuals from Zafferana and Bajna carried $w$ Cer1 without $w$ Cer2 [13]. Furthermore, individuals from these populations may also carry $w$ Cer $3, w$ Cer 4 and $w$ Cer5 $[14,38]$.

In 2002, wCer4 was successfully established in an isofemale line of C. capitata Benakeion (WolMed S10.3, hereafter called Ccap10.3) by microinjection, using a donor population of $R$. cerasi from Sicily [69]. One Ccap10.3 individual from generation 30 was selected for DNA extraction and whole genome sequencing.

Previously, the genome of $w$ Cer2 [24] was assembled from sequencing reads derived from embryos of $D$. simulans isofemale line DsimRC50 carrying a single infection of $w$ Cer2, that had been established following embryonic microinjection from $R$. cerasi individuals from Schoenbrunn, Vienna [41]. The wCer2 genome was also obtained from another $D$. simulans isofemale line (DsimRC45), the RcerAS individual, and from an individual of another C. capitata isofemale line, Ccap88.6, which was established after microinjection of $w$ Cer2 from RcerAS individuals into C. capitata Benakeion individuals [24].

\section{DNA extraction and high-throughput DNA sequencing}

For $R$. cerasi and C. capitata, DNA was extracted from an individual larva (RcerIZ), pupa (RcerAS and RcerHB) or adult female abdomen (Ccap10.3), based on availability of source material. The samples were first surface sterilized by immersion in 5\% sodium hypochlorite for 1 min, followed by rinsing in triton- $x$ and multiple washes of water. The QiaAmp DNA kit was used to isolate genomic DNA from each sample, according to the manufacturer's instructions, including RNase treatment, with the exception that the final elution was using $50 \mu \mathrm{L}$ of nuclease-free water. Quality of genomic DNA was checked by gel electrophoresis. Whole genome amplification of 5 to $20 \mathrm{ng}$ genomic DNA using the Qiagen Repli-G midi kit was performed to increase the quantity and proportion of bacterial DNA in the sample. The amplified DNA was cleaned again using the QiaAmp kit and eluted in $50 \mu \mathrm{L}$ nuclease-free water.

The quality and yield of all DNA samples was ascertained by gel electrophoresis, Nanodrop spectroscopy and Qubit double-stranded DNA quantification system. Libraries for each sample were prepared with TruSeq PCR-free (350 bp insert) library kit (Illumina), using $1 \mu \mathrm{g}$ of input DNA, and the paired-end $(2 \times 125$ bp) libraries were sequenced on an Illumina HiSeq 2500 platform (NGS Facility, Western Sydney University).

\section{Bioinformatics}

The bioinformatics pipeline was implemented as described in Morrow, et al. [24], with minor modifications. CLC Genomics Workbench ver.12 (Qiagen) was used for quality control, de novo assembly, mapping and variant calling. In order to choose the best sample library to use for the assembly of each of the Wolbachia genomes $w$ Cer1, $w$ Cer 4 and $w$ Cer5, the trimmed reads for each library were mapped at $100 \%$ similarity to the $w s p$ gene and the five MLST sequences (gatB, $\operatorname{cox} A, h c p A, f b p A$ and $f t s Z$ ) that were previously obtained for these strains [14]. As the MLST profile for $w$ Cer3 is unknown [38] we used the $w$ Cer3 $w s p$ gene sequence to check for $w$ Cer3 reads. The library of the Ccap10.3 line only had reads mapping to the $w$ Cer4-specific $w s p$ and MLST gene sequences, in line with the expectation based on PCR-based analysis [42]. Mapping of read sequences of the RcerHB library to the $w$ Cer $w s p$ and MLST sequences showed that $w$ Cer1 was present as a single infection with no background reads for any other strain. RcerIZ mapped only to $w$ Cer 1 (moderate) and $w$ Cer 5 (low); and RcerAS mapped to $w$ Cer1 (moderate), $w$ Cer2 (high) and wCer5 (low) as stated in Morrow, et al. [24]. Therefore, Ccap10.3 was used to assemble $w$ Cer 4 , and RcerHB was used to assemble $w$ Cer1. By using parameters that filtered and excluded $w$ Cer 1 and $w$ Cer2 reads, RcerAS alone was used to assemble $w$ Cer5, because mapped reads of RcerIZ to the $w$ Cer5 contigs produced low coverage.

To generate the draft genomes of the supergroup A strains $w C e r 1$ and $w C e r 4$ found as single infections in the sequenced individuals, trimmed paired reads were de novo assembled into contigs using default parameters in CLC Genomics Workbench. Each set of contigs was queried using the complete Wolbachia genomes wMel (GenBank: AE017196), wRi (GenBank: CP001391) and $w$ Ha (GenBank: NC021089). Those contigs identified as Wolbachia sequence were extracted and aligned against 
$w$ Mel using Mauve [70]. The reordered contigs were manually scaffolded and GapFiller [71] extended the sequence and closed the gaps where possible. The $w$ Cer 1 and $w$ Cer 4 scaffolds were subsequently realigned with other reference sequences ( $w \mathrm{Ri}$ and $w \mathrm{Ha}$ ), and Gapfilling and mapping were repeated. The scaffolds were finally mapped at $99 \%$ similarity over $95 \%$ of the read length to verify the genome sequence.

For the supergroup B strain $w$ Cer5, assembled contigs from RcerAS were queried by the $w$ Pip (GenBank NC_ 010981) genome. Only the contigs larger than $500 \mathrm{bp}$ and with a match of above $95 \%$ were kept, to exclude contigs representing $w$ Cer1 or $w$ Cer 2 in that individual. Contigs were then arranged in order of the $w$ Pip genome using Mauve, and reads were mapped at a high stringency of $98 \%$ similarity and $90 \%$ length to again favour $w$ Cer5 reads. GapFiller could not be used because the highest proportion of reads in the RcerAS libraries was for $w$ Cer2 and $w$ Cer1, respectively, which were at times preferentially incorporated instead of $w$ Cer 5 sequences, and, therefore, introduced too many errors. RcerAS reads were mapped to $w$ Pip at a stringency of $97 \%$ for $60-90 \%$ of the read length to also extend the $w$ Cer 5 contig sequences. Any contigs at this stage that had excessively high relative coverage were identified, checked against the $w$ Cer 1 and $w$ Cer 2 genomes and removed if identical to $w$ Cer1 or $w$ Cer2. When polymorphic sequences were found, the alternatives were checked against the known assemblies of $w C e r 1$ and $w C e r 2$ and removed. This careful approach ensured that the $w$ Cer5 draft genome did not include sequences from $w$ Cer1 or $w$ Cer2. However, it is possible that the assembled $w$ Cer5 genome is missing some sequences that were removed, particularly if a region is identical or very similar to $w$ Cer 1 and $w$ Cer 2 sequences.

The final draft genomes were mapped at a stringency of $99 \%$ over $95 \%$ of the read length: reads from RcerHB were mapped to $w$ Cer1 (16 contigs); reads from Ccap10.3 were mapped to $w$ Cer4 $(65$ contigs) and reads from RcerAS were mapped to $w$ Cer5 (57 contigs).

\section{Annotation and analysis}

Each of the draft genomes $w$ Cer1 (RcerHB), $w$ Cer4 (Ccap10.3) and $w$ Cer5 (RcerAS) were submitted to NCBI. The three genomes plus 16 reference genomes were all annotated using PROKKA v1.13.3 [72] to standardise the subsequent analyses. The completeness of the new genomes was ascertained by comparison to other complete Wolbachia genomes via the BUSCO v3.0.2 pipeline for Proteobacteria, which determines the presence of a standardised set of 221 single copy genes in each genome [73]. Prophage regions were annotated using the PHASTER server [74].
OrthoFinder version 2.3.1 [75] was applied with default parameters to the coding sequences identified in $w$ Cer $1, w$ Cer 4 and $w$ Cer5 and an additional 16 reference genomes, listed in Table 2. Orthologous genes from these 19 genomes were clustered into orthogroups, and these groupings were visualised using the UpSetR package [76], and also supported multigene phylogenetic analysis and the identification of target gene orthologues, such as cifA and cifB orthologues.

A subset of coding sequences common to all 19 genomes was aligned and maximum likelihood phylogenetic trees were computed. The set of single copy orthologues identified in OrthoFinder were further filtered for recombinant loci as previously described [24] using PhiPack [77]. Gene (codon) alignments, and subsequent determination of monophyletic adherence to supergroup A and B classifications, were performed in $\mathrm{R}$ using the ape package [78]. Maximum likelihood trees were estimated using IQ-TREE [79] from concatenated gene alignments using FASconCAT [80] and a general time reversible base substitution model $(\mathrm{GTR}+\mathrm{F}+\mathrm{R} 2)$ as selected by ModelFinder [81].

Orthologues of cifA and cifB genes were found by locating orthogroups containing the cifA and cifB genes from $w$ Mel [T1], $w$ No [T3], $w$ Pip [T4] and $w$ Stri [T5]. The nomenclature of cif gene pairs has recently been proposed to follow the format of $c i f_{w S t r a i n[T 1]}$ as an example for a Type I pair [82]. Protein domains within CifA and CifB were identified by HHPred, using databases SCOPe70_2.07, Pfam-A_v34, COG_KOG_v1.0 and SMART_v6.0 [83], furin cleavage sites were detected using PiTou [84] and the gene structures of CifA and CifB were prepared using IBS data visualisation [85]. The gene sequences were codon-aligned in MEGA v7 using Muscle with special consideration of the domains highlighted by HHPred, in Lindsey et al. [23] and the mutagenesis study by Shropshire et al. [54]. The cifA alignment excluded orthologues if they were truncated and did not contain the unannotated $\mathrm{N}$-terminal region, or the catalase-rel or DUF domains, because mutations in any of these essential regions can diminish $\mathrm{CI}$ and rescue [54]. The cifB gene alignment included orthologues only if they contained the unannotated $\mathrm{N}$-terminal region and the two PDDEXK domains common to Type I and Type IV cifB genes which have both been determined experimentally to induce CI. Maximum likelihood trees were estimated from the gene alignments as described above, using models TPM $3+\mathrm{F}+\mathrm{G} 4$ for cifA and $\mathrm{TPM} 3+\mathrm{F}+\mathrm{I}+\mathrm{G} 4$ for $c i f B$.

$w m k$ orthologues were identified in the $w$ Cer genomes from the orthogroup containing $w$ Mel WD0626 (wmk). No orthologues were found in $w$ Cer1, but each orthologue from $w$ Mel (seven genes), $w$ Rec (one gene), $w$ Cer2 (eight genes), $w$ Cer4 (five genes) and $w$ Cer5 (three 
genes) were codon-aligned in MEGA v7 using Muscle, and the amino acid pairwise distances were calculated.

\section{Variant analysis of wCer 1 and $w$ Cer5 genomes in different hosts}

Polymorphisms between $w$ Cer1 variants of three populations (RcerAS, RcerHB, RcerIZ), and between $w$ Cer5 variants of two populations (RcerAS, RcerIZ), were identified by read mapping using CLC Genomics Workbench to the final draft genomes at a similarity of $97 \%$ over $97 \%$ of the read length, and only properly paired reads were kept. These parameters differed from the parameters used to verify the draft genome sequences because here we wanted to capture strain variation within an individual. For the RcerAS library (comprising $w$ Cer1, $w$ Cer2 and $w$ Cer5), reads were competitively mapped to the $w$ Cer2 (GenBank Accession No: SOZK01000000) as well as the $w$ Cer 1 and $w$ Cer 5 genomes simultaneously to restrict errors, primarily due to $w$ Cer 2 reads mapping to the $w$ Cer1 genome. This problem was likely to occur because $w$ Cer 1 and $w$ Cer 2 are both supergroup A strains and $w$ Cer 2 reads were more abundant than $w$ Cer 1 reads and would therefore inflate the outcome of variant detection. The RcerIZ library was competitively mapped to $w$ Cer 1 and $w$ Cer5, and RcerHB was only mapped to $w$ Cer1. These stringency parameters allowed for polymorphisms to be detected, while minimising off target reads.

Read mapping was used to (i) generate a libraryspecific consensus sequence for each strain and (ii) detect variation within individuals for each strain. For $w$ Cer1 variant SNP calling, the RcerAS and RcerHB libraries were subsampled (26 million and 10 million reads respectively) to normalize the number of $w$ Cer1 reads against the full RcerIZ library that had 258,194 properly paired reads mapped to $w$ Cer1 (average $20 \mathrm{x}$ coverage). For $w$ Cer5 variant SNP calling, the RcerAS library was not subsampled to the level of RcerIZ, because the low number of reads from RcerIZ (51,402 reads) gave very low and sparse coverage (average $4 \mathrm{x})$. The full RcerAS library was used and provided $\sim 18 \mathrm{x}$ coverage. In order to aid analysis, variants of $w$ Cer2 from two D. simulans lines and one C. capitata line carrying single infections of $w$ Cer2 [24] were also analysed along with $w$ Cer2 from RcerAS subsampled to 87 million reads. To determine the consensus sequences, no minimum read number threshold was applied and the majority (>50\% reads) nucleotides were extracted for each Wolbachia strain derived from each library to determine a library specific genome. Alignment of these consensus sequences (Additional Files 7, 8 and 9) were used to draw variant genome networks for $w$ Cer $1, w$ Cer 2 and $w$ Cer 5 using Popart [86]. For variant detection within an individual, the threshold for read coverage was set at five reads, if fewer reads mapped the reference nucleotide was called as default. Variant detection was performed in CLC Genomics Workbench, with variant sites only listed (in Additional Files 10, 11 and 12) for SNPs present at a minimum of $35 \%$ of reads. These parameters were selected to highlight prevalent variation and minimise false positives due to sequence errors.

\section{Mitochondrial genomes}

The mitochondrial genome contigs were extracted from each $R$. cerasi de novo assembly via BLASTn match to the $C$. capitata complete mitochondrial genome (GenBank Acc: AJ242872). The sequencing reads were mapped at high stringency (97\% similarity and $90 \%$ length) and the circular genomes closed and verified by mapping at 99\% similarity and 95\% length. Protein coding genes (PCGs), tRNAs and rRNAs were annotated using Mitos2 [87] and manually adjusted in line with published annotations of other tephritid mitogenomes. Sequences were aligned in MEGA v7 using MUSCLE, and differences were noted.

SNPs across the three $R$. cerasi mitochondrial genomes were identified by mapping a subsample of each library to the RcerHB mitochondrial genome at $97 \%$ similarity and $97 \%$ length. Each library was subsampled to achieve approximately 500-fold coverage of the mitogenome, hence RcerHB (530,000 paired reads), RcerIZ $(12,000,000$ paired reads) and RcerAS (270,000 paired reads) were sampled and mapped to the RcerHB mitogenome. Variant SNPs were called with a low frequency cut-off of $1 \%$, and differences between populations were identified when found at $>99 \%$ frequency.

\section{Linkage of mitochondrial haplotypes and Wolbachia strains}

A ddRADseq dataset representing 192 R. cerasi individuals from six countries (Austria, Germany, Italy, Norway, Portugal and Iran) published as part of a population study [53] was downloaded from the NCBI SRA (Acc. No. SRX6787773). The 273,988,021 raw reads included the barcodes and the modified restriction site at the $5^{\prime}$ end of the sequence. These reads were competitively mapped to the four Wolbachia genomes (wCer1, wCer2, $w$ Cer4, $w$ Cer5) and the mitochondrial genome of RcerHB at $85 \%$ of read length and $98 \%$ similarity, and only reads specific to a single genome were retained. This low length stringency was chosen so the overhanging barcode of 8 to 10 nucleotides met the parameters. The barcodes were used to identify the samples that mapped to regions on the mitochondrial genome that showed variability. These samples were scored for $\mathrm{Wol}$ bachia strain presence by examining mapping coverage 
over the four Wolbachia genomes. The threshold selected was at least one perfect read over at least five mapped regions of the genome. This threshold meant that low titre strains were reliably detected (even at onefold coverage over many regions) but eliminated misallocation of reads to a different Wolbachia strain where the genome was incomplete; this could have occurred if $w$ Cer5 was present but the conservative approach to its genome assembly resulted in some of its reads mapping to another genome.

Mitochondrial SNPs for each of 46 samples were identified from the ddRadseq mapped reads, tabulated and converted to a genind object and a Euclidean distance matrix using adegenet $[88,89]$ in $\mathrm{R}[90]$. Samples were grouped as HT1 or HT2, based on presence or absence of $w$ Cer2, if they had $w$ Cer 4 or $w$ Cer 5 , and by country of origin (with Sicily divided into Sicily West and Sicily East). Adonis of the $\mathrm{R}$ package vegan [91] was implemented to perform a PERMANOVA to detect differences between the groups.

\section{Supplementary Information}

The online version contains supplementary material available at https://doi. org/10.1186/s12864-021-07906-6.

Additional file 1. UpSet graph showing shared Wolbachia orthogroups. Additional file 2. PHASTER identification of prophage regions in wCer1, wCer4 and wCer5.

Additional file 3. cifA and cifB locus names from Wolbachia strains present in phylogeny.

Additional file 4. Mitochondrial genomes and the polymorphic sites between RcerHB (HT1a), RcerlZ (HT1b) and RcerAS (HT2).

Additional file 5. Polymorphisms in mitogenomes of three complete mitogenomes of RcerHB (HT1a), RcerlZ (HT1b) and RcerAS (HT2) and an additional 46 samples with SNP representation at 12 of these poymorphic sites from ddRadSeq data. Sample names and location are from Bakovic et al [53], together with associated data.

Additional file 6. PERMANOVA of mitochondrial genetic distances between samples grouped by presence of Wolbachia strains.

Additional file 7. FASTA aligned $w$ Cer1 consensus sequences from RcerHB, RcerlZ and RcerAS.

Additional file 8. FASTA aligned $w$ Cer 5 consensus sequences from RcerlZ and RcerAS.

Additional file 9. FASTA aligned wCer2 consensus sequences from RcerAS, DsimRC45, DsimRC50 and Ccap88.6.

Additional file 10. $w C e r 1$ variant calling. Reference position refers to the wCer1 genome position after the 16 contigs were joined in order Reads from three libraries: RcerHB (sampled to 10 million reads), RcerlZ (all reads) and RcerAS (sampled to 26 milion reads), were mapped at 97\% length and $97 \%$ similarity, and RcerlZ and RcerAS were mapped competitively to the genomes of wCer 1 and wCer5; and wCer1, wCer2 and $w$ Cer5, respectively. Variants were called with a minimum cut-off of $35 \%$ frequency, so a frequency of $65 \%$ for a variant is considered homozygous. Location of SNPS within genes is based on PROKKA annotation, and determination of copy number (single or multiple copy) was based on Orthofinder assessment of orthogroups including wCer1, wAu and wMel genomes.

Additional file 11. $w$ Cer 5 variant calling. Reference position refers to the wCer 5 genome position after the 57 contigs were joined in order Reads from two libraries: RcerlZ (all reads) and RcerAS (all reads), were mapped at 97\% length and 97\% similarity, and RcerlZ and RcerAS were mapped competitively to the genomes of wCer 1 and $w C e r 5$, and wCer 1 , wCer2 and wCer5, respectively. Variants were called with a minimum cutoff of $35 \%$ so a frequency of $65 \%$ for a variant is considered homozygous. Location of SNPs within genes is based on PROKKA annotation, and determination of copy number (single or multiple copy) was based on Orthofinder assessment of orthogroups including wCer5, wMeg and wPip genomes.

Additional file 12. wCer 2 variant calling. Reference position refers to the wCer 2 genome position after the 11 contigs were joined in order. RcerAS (sampled to 87 million reads) was mapped competitively to the genomes of $w$ Cer1, wCer2 and wCer5, respectively at 97\% length and $97 \%$ similarity. Variants were called with a minimum cut-off of $35 \%$ frequency.

\section{Acknowledgements}

We thank K. Bourtzis for supply of C. capitata individuals of the lines Ccap10.3 and Ccap88.6, C. Janitz for technical advice and support with NGS sequencing of libraries, and G. Devine for IT support.

\section{Authors' contributions}

JLM conceived the study, generated, analysed, and interpreted the data and wrote the manuscript. MR conceived the study, collected the material, interpreted data and wrote the manuscript. All authors read and approved the final manuscript.

\section{Funding}

This research was supported by the Australian Government through the Australian Research Council (ARC) Industrial Transformation Training Centre (ITTC) Fruit Fly Biosecurity Innovation (IC150100026), the Hawkesbury Institute for the Environment and Western Sydney University. The funding bodies had no role in the design of the study, or in the collection, analysis, and interpretation of data, or in writing this manuscript.

\section{Availability of data and materials}

The wCer1, wCer4 and wCer5 genomes were submitted as a Whole Genome Shotgun project (BioProject No. PRJNA668868) at DDBJ/ENA/GenBank under the accessions JADCNC000000000 (wCer1) (https://www.ncbi.nlm.nih.gov/ nuccore/JADCNC000000000); JADCND000000000 (wCer4) (https://www.ncbi. nlm.nih.gov/nuccore/JADCND000000000); and JADCNE0000000000 (wCer5) (https://www.ncbi.nlm.nih.gov/nuccore/JADCNE000000000). The versions described in this paper are JADCNC010000000, JADCND010000000 and JADCNE0100000000. Raw reads were submitted to NCBI SRA (sequence read archive), also under BioProject No. PRJNA668868 (https://www.ncbi.nlm.nih. gov/sra/PRJNA668868).

\section{Declarations}

Ethics approval and consent to participate

Not applicable.

\section{Consent for publication}

Not applicable.

\section{Competing interests}

The authors declare that they have no competing interests.

Received: 19 May 2021 Accepted: 20 July 2021

Published online: 13 August 2021

References

1. Werren JH, Baldo L, Clark ME. Wolbachia: master manipulators of invertebrate biology. Nat Rev Microbiol. 2008;6(10):741-51. https://doi.org/1 $0.1038 /$ nrmicro1969.

2. Kaur R, Shropshire JD, Cross KL, Leigh B, Mansueto AJ, Stewart V, et al. Living in the endosymbiotic world of Wolbachia: a centennial review. Cell Host Microbe. 2021;29(6):879-93. https://doi.org/10.1016/j.chom.2021.03.006.

3. Hurst GDD, Jiggins FM. Problems with mitochondrial DNA as a marker in population, phylogeographic and phylogenetic studies: the effects of 
inherited symbionts. Proc R Soc Lond B Biol Sci. 2005;272(1572):1525-34. https://doi.org/10.1098/rspb.2005.3056.

4. Hurst GD, Frost CL. Reproductive parasitism: maternally inherited symbionts in a biparental world. Cold Spring Harb Perspect Biol. 2015;7(5):a017699. https://doi.org/10.1101/cshperspect.a017699.

5. Doremus MR, Hunter MS. The saboteur's tools: common mechanistic themes across manipulative symbioses. Adv Insect Physiol. 2020;58:317-53. https://doi.org/10.1016/bs.aiip.2020.03.003.

6. Hosokawa T, Koga R, Kikuchi Y, Meng XY, Fukatsu T. Wolbachia as a bacteriocyte-associated nutritional mutualist. Proc Natl Acad Sci U S A. 2010; 107(2):769-74. https://doi.org/10.1073/pnas.0911476107.

7. Brownlie JC, Cass BN, Riegler M, Witsenburg JJ, Iturbe-Ormaetxe I, McGraw EA, et al. Evidence for metabolic provisioning by a common invertebrate endosymbiont, Wolbachia pipientis, during periods of nutritional stress. PLoS Pathog. 2009;5(4):e1000368. https://doi.org/10.1371/journal.ppat.1000368.

8. Weeks AR, Turelli M, Harcombe WR, Reynolds KT, Hoffmann AA. From parasite to mutualist: rapid evolution of Wolbachia in natural populations of Drosophila. PLoS Biol. 2007;5(5):e1 14. https://doi.org/10.1371/journal.pbio. 0050114.

9. Teixeira L, Ferreira Á, Ashburner M. The bacterial symbiont Wolbachia induces resistance to RNA viral infections in Drosophila melanogaster. PLoS Biol. 2008;6(12):e1000002. https://doi.org/10.1371/journal.pbio.1000002.

10. Hedges LM, Brownlie JC, O'Neill SL, Johnson KN. Wolbachia and virus protection in insects. Science. 2008;322(5902):702. https://doi.org/10.1126/ science.1162418.

11. Hoffmann AA, Turelli M. Unidirectional incompatibility in Drosophila simulans: inheritance, geographic variation and fitness effects. Genetics. 1988;119(2):435-44. https://doi.org/10.1093/genetics/119.2.435.

12. Min KT, Benzer S. Wolbachia, normally a symbiont of Drosophila, can be virulent, causing degeneration and early death. Proc Natl Acad Sci U S A. 1997;94(20):10792-6. https://doi.org/10.1073/pnas.94.20.10792.

13. Riegler M, Stauffer C. Wolbachia infections and superinfections in cytoplasmically incompatible populations of the European cherry fruit fly Rhagoletis cerasi (Diptera, Tephritidae). Mol Ecol. 2002;11(11):2425-34. https://doi.org/10.1046/j.1365-294x.2002.01614.x.

14. Arthofer W, Riegler M, Schneider D, Krammer M, Miller WJ, Stauffer C. Hidden Wolbachia diversity in field populations of the European cherry fruit fly, Rhagoletis cerasi (Diptera, Tephritidae). Mol Ecol. 2009;18(18):3816-30. https://doi.org/10.1111/j.1365-294X.2009.04321.x.

15. Merçot $\mathrm{H}$, Charlat $\mathrm{S}$. Wolbachia infections in Drosophila melanogaster and $D$. simulans: polymorphism and levels of cytoplasmic incompatibility. Drosophila melanogaster, Drosophila simulans: So Similar, So Different; 2004. p. 51-9.

16. Perrot-Minnot MJ, Guo LR, Werren JH. Single and double infections with Wolbachia in the parasitic wasp Nasonia vitripennis: effects on compatibility. Genetics. 1996;143(2):961-72. https://doi.org/10.1093/genetics/143.2.961.

17. Bordenstein SR, O'Hara FP, Werren JH. Wolbachia-induced incompatibility precedes other hybrid incompatibilities in Nasonia. Nature. 2001;409(6821): 707-10. https://doi.org/10.1038/35055543.

18. Bordenstein $\mathrm{SR}$, Werren JH. Bidirectional incompatibility among divergent Wolbachia and incompatibility level differences among closely related Wolbachia in Nasonia. Heredity. 2007;99(3):278-87. https://doi.org/10.1038/sj. hdy.6800994.

19. Wade MJ. Infectious speciation. Nature. 2001;409(6821):675-7. https://doi. org/10.1038/35055648.

20. LePage DP, Metcalf JA, Bordenstein SR, On J, Perlmutter JI, Shropshire JD, et al. Prophage WO genes recapitulate and enhance Wolbachia-induced cytoplasmic incompatibility. Nature. 2017;543(7644):243-7. https://doi.org/1 0.1038 /nature21391.

21. Beckmann JF, Ronau JA, Hochstrasser M. A Wolbachia deubiquitylating enzyme induces cytoplasmic incompatibility. Nat Microbiol. 2017;2(5):17007. https://doi.org/10.1038/nmicrobiol.2017.7.

22. Shropshire JD, Bordenstein SR. Two-by-one model of cytoplasmic incompatibility: synthetic recapitulation by transgenic expression of cifA and cifB in Drosophila. PLoS Genet. 2019;15(6):e1008221. https://doi.org/10.1371/ journal.pgen.1008221.

23. Lindsey ARI, Rice DW, Bordenstein SR, Brooks AW, Bordenstein SR, Newton ILG. Evolutionary genetics of cytoplasmic incompatibility genes cifA and cifB in prophage WO of Wolbachia. Genome Biol Evol. 2018;10(2):434-51. https://doi.org/10.1093/gbe/evy012.

24. Morrow JL, Schneider DI, Klasson L, Janitz C, Miller WJ, Riegler M. Parallel sequencing of Wolbachia wCer2 from donor and novel hosts reveals multiple incompatibility factors and genome stability after host transfers. Genome Biol Evol. 2020;12(5):720-35. https://doi.org/10.1093/ gbe/evaa050

25. Madhav M, Parry R, Morgan JAT, James P, Asgari S. Wolbachia endosymbiont of the horn fly Haematobia irritans irritans: a supergroup A strain with multiple horizontally acquired cytoplasmic incompatibility genes. Appl Environ Microbiol. 2020;86(6):e02589-19.

26. Martinez J, Klasson L, Welch JJ, Jiggins FM. Life and death of selfish genes: comparative genomics reveals the dynamic evolution of cytoplasmic incompatibility. Mol Biol Evol. 2020;38(1):2-15.

27. Bing XL, Zhao DS, Sun JT, Zhang KJ, Hong XY. Genomic analysis of Wolbachia from Laodelphax striatellus (Delphacidae, Hemiptera) reveals insights into its "Jekyll and Hyde" mode of infection pattern. Genome Biol Evol. 2020;12(2):3818-31. https://doi.org/10.1093/gbe/evaa006.

28. Shropshire JD, Rosenberg R, Bordenstein SR. The impacts of cytoplasmic incompatibility factor (cifA and cifB) genetic variation on phenotypes. Genetics. 2021;217(1):iyaa007.

29. Mercot H, Llorente B, Jacques M, Atlan A, Montchamp-Moreau C. Variability within the Seychelles cytoplasmic incompatibility system in Drosophila simulans. Genetics. 1995;141(3):1015-23. https://doi.org/10.1093/ genetics/141.3.1015.

30. Ellegaard KM, Klasson L, Naslund K, Bourtzis K, Andersson SG. Comparative genomics of Wolbachia and the bacterial species concept. PLoS Genet. 2013;9(4):e1003381. https://doi.org/10.1371/journal.pgen.1003381.

31. Noda H. Cytoplasmic incompatibility in a rice planthopper. J Hered. 1984; 75(5):345-8. https://doi.org/10.1093/oxfordjournals.jhered.a109953.

32. Bonneau M, Caputo B, Ligier A, Caparros R, Unal S, Perriat-Sanguinet M, et al. Variation in Wolbachia cidB gene, but not cidA, is associated with cytoplasmic incompatibility mod phenotype diversity in Culex pipiens. Mol Ecol. 2019;28(00):4725-36. https://doi.org/10.1111/mec.15252.

33. Sicard M, Namias A, Perriat-Sanguinet M, Carron E, Unal S, Altinli M, et al. Cytoplasmic incompatibility variations in relation with Wolbachia cid genes divergence in Culex pipiens. mBio. 2021;12(1):e02797-20.

34. Boller EF, Bush GL. Evidence for genetic variation in populations of the European cherry fruit fly, Rhagoletis cerasi (Diptera: Tephritidae) based on physiological parameters and hybridization experiments. Entomol Exp Appl. 1974;17(2):279-93. https://doi.org/10.1111/j.1570-7458.1974.tb00345.x.

35. Boller EF, Russ K, Vallo V, Bush GL. Incompatible races of European cherry fruit fly, Rhagoletis cerasi (Diptera: Tephritidae), their origin and potential use in biological control. Entomol Exp Appl. 1976;20(3):237-47. https://doi.org/1 0.1111/j.1570-7458.1976.tb02640.x.

36. Blümel S, Keck M, Nowotny N, Fiedler W, Russ K. Detection and therapy of Rickettsia-like-organisms (RLO's) in ovaries of the European cherry fruit fly (Rhagoletis cerasi L.; Trypetidae): an evaluation of their influence on the unidirectional crossing sterility. Pflanzenschutzberichte. 1991;52:41-7.

37. Schuler H, Köppler K, Daxböck-Horvath S, Rasool B, Krumböck S, Schwarz D, et al. The hitchhiker's guide to Europe: the infection dynamics of an ongoing Wolbachia invasion and mitochondrial selective sweep in Rhagoletis cerasi. Mol Ecol. 2016;25(7):1595-609. https://doi.org/10.1111/mec.13571.

38. Arthofer W, Riegler M, Schuler H, Schneider D, Moder K, Miller WJ, et al. Allele intersection analysis: a novel tool for multi locus sequence assignment in multiply infected hosts. PLoS One. 2011;6(7):e22198. https:// doi.org/10.1371/journal.pone.0022198.

39. Vallo V, Remund U, Boller E. Storage conditions of stockpiled diapausing pupae of Rhagoletis cerasi for obtaining high emergence rates. Entomophaga. 1976;21 (3):251-6. https://doi.org/10.1007/BF02371758.

40. Köppler K, Kaffer T, Vogt H. Substantial progress made in the rearing of the European cherry fruit fly, Rhagoletis cerasi. Entomol Exp Appl. 2009;132(3): 283-8. https://doi.org/10.1111/j.1570-7458.2009.00889.x.

41. Riegler M, Charlat S, Stauffer C, Mercot H. Wolbachia transfer from Rhagoletis cerasi to Drosophila simulans: investigating the outcomes of host-symbiont coevolution. Appl Environ Microbiol. 2004;70(1):273-9. https://doi.org/10.112 8/AEM.70.1.273-279.2004.

42. Zabalou S, Riegler M, Theodorakopoulou M, Stauffer C, Savakis C, Bourtzis K. Wolbachia-induced cytoplasmic incompatibility as a means for insect pest population control. Proc Natl Acad Sci U S A. 2004;101(42):15042-5. https:// doi.org/10.1073/pnas.0403853101.

43. Apostolaki A, Livadaras I, Saridaki A, Chrysargyris A, Savakis C, Bourtzis K. Transinfection of the olive fruit fly Bactrocera oleae with Wolbachia: towards a symbiont-based population control strategy. J Appl Entomol. 2011;135(7): 546-53. https://doi.org/10.1111/j.1439-0418.2011.01614.X. 
44. Wolfe TM, Bruzzese DJ, Klasson L, Corretto E, Lečić S, Stauffer C, et al. Comparative genome sequencing reveals insights into the dynamics of Wolbachia in native and invasive cherry fruit flies. Mol Ecol. 2021. https://doi. org/10.1111/mec.15923.

45. Riegler M. The endosymbiont Wolbachia (Alphaproteobacteria) in the European cherry fruit fly Rhagoletis cerasi (Diptera: Tephritidae): population dynamics and influence on population genetics. Vienna: BOKU - University of Natural Resources and Life Sciences; 2002.

46. Bakovic V, Schebeck M, Telschow A, Stauffer C, Schuler H. Spatial spread of Wolbachia in Rhagoletis cerasi populations. Biol Lett. 2018;14(5):20180161. https://doi.org/10.1098/rsbl.2018.0161.

47. Bakovic V, Schebeck M, Stauffer C, Schuler H. Wolbachia-mitochondrial DNA associations in transitional populations of Rhagoletis cerasi. Insects. 2020; 11(10):675. https://doi.org/10.3390/insects11100675.

48. Jaenike J, Dyer KA. No resistance to male-killing Wolbachia after thousands of years of infection. J Evol Biol. 2008;21(6):1570-7. https://doi.org/10.1111/ j.1420-9101.2008.01607.x.

49. Duplouy A, Hurst GDD, O'Neill SL, Charlat S. Rapid spread of male-killing Wolbachia in the butterfly Hypolimnas bolina. J Evol Biol. 2010;23(1):231-5. https://doi.org/10.1111/j.1420-9101.2009.01891.x.

50. Perlmutter Jl, Bordenstein SR, Unckless RL, LePage DP, Metcalf JA, Hill T, et al. The phage gene $w m k$ is a candidate for male killing by a bacterial endosymbiont. PLoS Pathog. 2019;15(9):e1007936. https://doi.org/10.1371/ journal.ppat.1007936.

51. Veneti Z, Zabalou S, Papafotiou G, Paraskevopoulos C, Pattas S, Livadaras I, et al. Loss of reproductive parasitism following transfer of male-killing Wolbachia to Drosophila melanogaster and Drosophila simulans. Heredity. 2012;109(5):306-12. https://doi.org/10.1038/hdy.2012.43.

52. Hornett EA, Charlat S, Duplouy AMR, Davies N, Roderick GK, Wedell N, et al. Evolution of male-killer suppression in a natural population. PLOS Biol. 2006; 4(9):1643-8

53. Bakovic V, Schuler H, Schebeck M, Feder JL, Stauffer C, Ragland GJ. Host plant-related genomic differentiation in the European cherry fruit fly, Rhagoletis cerasi. Mol Ecol. 2019;28(20):4648-66. https://doi.org/10.1111/ mec.15239.

54. Shropshire JD, Kalra M, Bordenstein SR. Evolution-guided mutagenesis of the cytoplasmic incompatibility proteins: Identifying CifA's complex functional repertoire and new essential regions in CifB. PLoS Pathog. 2020; 16(8):e1008794.

55. Kriesner $\mathrm{P}$, Conner WR, Weeks AR, Turelli M, Hoffmann AA. Persistence of a Wolbachia infection frequency cline in Drosophila melanogaster and the possible role of reproductive dormancy. Evolution. 2016;70(5):979-97. https://doi.org/10.1111/evo.12923.

56. Yamada R, Floate KD, Riegler M, O'Neill SL. Male development time influences the strength of Wolbachia-induced cytoplasmic incompatibility expression in Drosophila melanogaster. Genetics. 2007;177(2):801-8. https:// doi.org/10.1534/genetics.106.068486.

57. Hoffmann AA, Clancy D, Duncan J. Naturally-occurring Wolbachia infection in Drosophila simulans that does not cause cytoplasmic incompatibility. Heredity. 1996;76(1):1-8. https://doi.org/10.1038/hdy.1996.1.

58. Kriesner P, Hoffmann AA, Lee SF, Turelli M, Weeks AR. Rapid sequential spread of two Wolbachia variants in Drosophila simulans. PLoS Pathog. 2013; 9(9):e1003607. https://doi.org/10.1371/journal.ppat.1003607.

59. Cattel J, Nikolouli K, Andrieux T, Martinez J, Jiggins F, Charlat S, et al. Back and forth Wolbachia transfers reveal efficient strains to control spotted wing Drosophila populations. J Appl Ecol. 2018;55(5):2408-18. https://doi.org/1 $0.1111 / 1365-2664.13101$

60. Liang X, Liu J, Bian G, Xi Z. Wolbachia inter-strain competition and inhibition of expression of cytoplasmic incompatibility in mosquito. Front Microbiol. 2020;11:1638. https://doi.org/10.3389/fmicb.2020.01638

61. Chrostek E, Marialva MSP, Esteves SS, Weinert LA, Martinez J, Jiggins FM, et al. Wolbachia variants induce differential protection to viruses in Drosophila melanogaster: a phenotypic and phylogenomic analysis. PLoS Genet. 2013;9(12):e1003896. https://doi.org/10.1371/journal.pgen.1003896 .

62. Chen H, Ronau JA, Beckmann JF, Hochstrasser M. A Wolbachia nuclease and its binding partner provide a distinct mechanism for cytoplasmic incompatibility. Proc Natl Acad Sci. 2019;116(44):22314-21. https://doi.org/1 0.1073/pnas.1914571116.

63. Zabalou S, Apostolaki A, Pattas S, Veneti Z, Paraskevopoulos C, Livadaras I, et al. Multiple rescue factors within a Wolbachia strain. Genetics. 2008;178(4): 2145-60. https://doi.org/10.1534/genetics.107.086488.
64. López-Madrigal S, Duarte EH. Titer regulation in arthropod-Wolbachia symbioses. FEMS Microbiol Lett. 2019;366(23):fnz232.

65. Layton EM, On J, Perlmutter JI, Bordenstein SR, Shropshire JD. Paternal grandmother age affects the strength of Wolbachia-induced cytoplasmic incompatibility in Drosophila melanogaster. MBio. 2019; 10(6):e01879-19.

66. Reynolds KT, Hoffmann AA. Male age, host effects and the weak expression or non-expression of cytoplasmic incompatibility in Drosophila strains infected by maternally transmitted Wolbachia. Genet Res. 2002;80(2):79-87. https://doi.org/10.1017/S0016672302005827.

67. Ruang-areerate T, Kittayapong P, McGraw EA, Baimai V, O’Neill SL. Wolbachia replication and host cell division in Aedes albopictus. Curr Microbiol. 2004; 49(1):10-2. https://doi.org/10.1007/s00284-003-4245-8.

68. Schuler H, Bertheau C, Egan SP, Feder JL, Riegler M, Schlick-Steiner BC, et al. Evidence for a recent horizontal transmission and spatial spread of Wolbachia from endemic Rhagoletis cerasi (Diptera: Tephritidae) to invasive Rhagoletis cingulata in Europe. Mol Ecol. 2013;22(15):4101-11. https://doi. org/10.1111/mec.12362.

69. Zabalou S, Apostolaki A, Livadaras I, Franz G, Robinson AS, Savakis C, et al. Incompatible insect technique: incompatible males from a Ceratitis capitata genetic sexing strain. Entomol Exp Appl. 2009;132(3):232-40. https://doi. org/10.1111/j.1570-7458.2009.00886.x.

70. Darling AC, Mau B, Blattner FR, Perna NT. Mauve: multiple alignment of conserved genomic sequence with rearrangements. Genome Res. 2004; 14(7):1394-403. https://doi.org/10.1101/gr.2289704.

71. Boetzer M, Pirovano W. Toward almost closed genomes with GapFiller. Genome Biol. 2012;13(6):R56. https://doi.org/10.1186/gb-2012-13-6-r56.

72. Seemann T. Prokka: rapid prokaryotic genome annotation. Bioinformatics. 2014;30(14):2068-9. https://doi.org/10.1093/bioinformatics/btu153.

73. Simão FA, Waterhouse RM, loannidis P, Kriventseva EV, Zdobnov EM. BUSCO: assessing genome assembly and annotation completeness with single-copy orthologs. Bioinformatics. 2015;31(19):3210-2. https://doi.org/1 0.1093/bioinformatics/btv351

74. Arndt D, Grant JR, Marcu A, Sajed T, Pon A, Liang Y, et al. PHASTER: a better, faster version of the PHAST phage search tool. Nucleic Acids Res. 2016; 44(W1):W16-21. https://doi.org/10.1093/nar/gkw387.

75. Emms DM, Kelly S. OrthoFinder: solving fundamental biases in whole genome comparisons dramatically improves orthogroup inference accuracy. Genome Biol. 2015;16(1):157. https://doi.org/10.1186/s13059015-0721-2.

76. Conway JR, Lex A, Gehlenborg N. UpSetR: an R package for the visualization of intersecting sets and their properties. Bioinformatics. 2017;33(18):2938-40. https://doi.org/10.1093/bioinformatics/btx364

77. Bruen TC, Philippe H, Bryant D. A simple and robust statistical test for detecting the presence of recombination. Genetics. 2006;172(4):2665-81. https://doi.org/10.1534/genetics.105.048975.

78. Paradis E, Schliep K. Ape 5.0: an environment for modern phylogenetics and evolutionary analyses in R. Bioinformatics. 2019;35(3):526-8. https://doi.org/1 0.1093/bioinformatics/bty633.

79. Nguyen L-T, Schmidt HA, von Haeseler A, Minh BQ. IQ-TREE: a fast and effective stochastic algorithm for estimating maximum-likelihood phylogenies. Mol Biol Evol. 2014;32(1):268-74. https://doi.org/10.1093/ molbev/msu300.

80. Kück P, Meusemann K. FASconCAT: convenient handling of data matrices. Mol Phylogenet Evol. 2010;56(3):1115-8. https://doi.org/10.1016/j.ympev.201 0.04.024.

81. Kalyaanamoorthy S, Minh BQ, Wong TKF, von Haeseler A, Jermiin LS. ModelFinder: fast model selection for accurate phylogenetic estimates. Nat Methods. 2017;14(6):587-9. https://doi.org/10.1038/nmeth.4285.

82. Shropshire JD, Leigh B, Bordenstein SR. Symbiont-mediated cytoplasmic incompatibility: what have we learned in 50 years? eLife. 2020;9:e61989. https://doi.org/10.7554/eLife.61989.

83. Söding J, Biegert A, Lupas AN. The HHpred interactive server for protein homology detection and structure prediction. Nucleic Acids Res. 2005; 33(Web Server issue):W244-8.

84. Tian S, Huajun W, Wu J. Computational prediction of furin cleavage sites by a hybrid method and understanding mechanism underlying diseases. Sci Rep. 2012;2(1):261. https://doi.org/10.1038/srep00261.

85. Liu W, Xie Y, Ma J, Luo X, Nie P, Zuo Z, et al. IBS: an illustrator for the presentation and visualization of biological sequences. Bioinformatics. 2015; 31(20):3359-61. https://doi.org/10.1093/bioinformatics/btv362. 
86. Leigh JW, Bryant D. popart: full-feature software for haplotype network construction. Methods Ecol Evol. 2015;6(9):1110-6. https://doi.org/10.1111/2 041-210X.12410.

87. Bernt $M$, Donath $A$, Jühling $F$, Externbrink F, Florentz C, Fritzsch G.

MITOS: improved de novo metazoan mitochondrial genome annotation. Mol Phylogenet Evol. 2013;69(2):313-9. https://doi.org/10.1016/j.ympev.2 012.08.023.

88. Jombart T. Adegenet: a R package for the multivariate analysis of genetic markers. Bioinformatics. 2008;24(11):1403-5. https://doi.org/10.1093/ bioinformatics/btn129.

89. Jombart T, Ahmed I. Adegenet 1.3-1: new tools for the analysis of genomewide SNP data. Bioinformatics. 2011;27(21):3070-1. https://doi.org/10.1093/ bioinformatics/btr521.

90. R Core Team: R. A language and environment for statistical computing. Vienna: R Foundation for statistical Computing; 2020

91. Oksanen J, Blanchet FG, Friendly M, Kindt R, Legendre P, McGlinn D, Minchin PR, O'Hara RB, Simpson GL, Solymos P et al: vegan: community ecology package. 2019

\section{Publisher's Note}

Springer Nature remains neutral with regard to jurisdictional claims in published maps and institutional affiliations.

Ready to submit your research? Choose BMC and benefit from:

- fast, convenient online submission

- thorough peer review by experienced researchers in your field

- rapid publication on acceptance

- support for research data, including large and complex data types

- gold Open Access which fosters wider collaboration and increased citations

- maximum visibility for your research: over $100 \mathrm{M}$ website views per year

At BMC, research is always in progress.

Learn more biomedcentral.com/submissions 